\title{
Consensusabilization for Continuous-Time High-Order Multiagent Systems with Time-Varying Delays
}

\author{
Huayong Zhu, ${ }^{1}$ Yirui Cong, ${ }^{1}$ Xiangke Wang, ${ }^{1}$ Daibing Zhang, ${ }^{1}$ and Qingjie Zhang ${ }^{2}$ \\ ${ }^{1}$ College of Mechatronics and Automation, National University of Defense Technology, Changsha 410073, China \\ ${ }^{2}$ Control Department, Aviation University of Air Force, Changchun 130022, China \\ Correspondence should be addressed to Xiangke Wang; xkwang@nudt.edu.cn
}

Received 6 May 2013; Revised 4 July 2013; Accepted 25 July 2013

Academic Editor: Trung Nguyen Thoi

Copyright (c) 2013 Huayong Zhu et al. This is an open access article distributed under the Creative Commons Attribution License, which permits unrestricted use, distribution, and reproduction in any medium, provided the original work is properly cited.

\begin{abstract}
For the consensus problems of high-order linear multiagent systems with time-varying delays in directed topologies, the LMI based-consensus criterion and NLMI-based consensusabilization (protocol parameters design that makes the multiagent systems achieve consensus) are investigated. Improved Lyapunov-Krasovskii functional is used for establishing the consensus convergence criteria and deriving the corresponding consensus protocol. In order to reduce the conservativeness, some proper free-weighting matrices are added into the derivative of Lyapunov-Krasovskii functional and that only keeps one necessary zoom. The numerical and simulation examples are given to demonstrate the effectiveness of the theoretical results. Compared with existing literatures, the convergence criterion and protocol design proposed have lower conservativeness.
\end{abstract}

\section{Introduction}

Recently, much attention is drawn to the consensus problem in multiagent systems [1-3], due to its widely applications ranging from rendezvous [4], flocking [5], formation control [6] to fusion estimation [7]. Furthermore, communication delays in consensus problems are also taken into account, for example, due to the communication congestions or retransmissions.

The study for consensus in multiagent systems with time delays started at first-order integrator systems (e.g., [8$14]$ ), some of which were generalized to second-order (e.g., [15-23]) or high-order (e.g., [24]) integrator systems then. Nevertheless, the system matrix in those systems is a special companion form matrix. Therefore, it is valuable to study the consensus problems of high-order (some paper called it high dimensional) linear systems with time-varying delays.

Compared with the consensus studies on first-order, second-order, and high-order integrator multiagent systems with time delays, relatively few results were conducted in those governed by high-order linear dynamics. For linear SISO multiagent systems, the robust consensus schemes with three kinds of feedback delays were investigated in [25]; and the necessary and sufficient condition for the existence of consensus solution for heterogeneous high-order multiagent systems was given in [26]. It should be noted that both of the above-mentioned studies were conducted in the frequency domain. In terms of linear MIMO multiagent systems, the time-domain methods were employed to analyze the consensus problems. In [27], the high-order linear MIMO consensus problems with single constant time delay under undirected communication topologies were converted into low-dimensional robust stability problems with time delay. The results showed that if the delay was less than a certain bound, the consensus protocols always existed; while [27] only gave the existence criteria on designing protocol parameters instead of algorithms for finding them. By projecting the state onto two subspaces (consensus subspace and consensus complement subspace), high-order linear MIMO consensus problems with single time-varying delay were equivalent simultaneous stabilization problems with low dimensions in [28]. Therefore, a Lyapunov-Krasovskii functional was given to establish both the consensus convergence and the algorithm for protocol parameters design [28]. However, 
there are still three main aspects that could be enhanced. (1) Conservativeness. The criterion had relatively more conservativeness caused by the structure of the Lyapunov-Krasovskii functional and the zoom in the derivative of this functional. (2) Constraint. All the derivatives $\mu$ of time delays were restricted by $\mu<1$ in [28]. (3) Parameters. The method of protocol design (the protocol has two parameter matrices and sometimes both of them need to be designed at once) could only calculate the second parameter matrix rather than both parameter matrices.

In this paper, we will provide free-weighting matrices [29] based consensus convergence criterion in high-order linear multiagent systems with single time-varying delay under directed topologies. Correspondingly, the protocol parameter matrices will be designed by solving NLMIs (nonlinear matrix inequalities). It should be noted that in undirected communicated topologies, the parameter matrices can be designed without constraining the structures for the solutions on NLMIs; while in directed topologies, some parts of those solutions should be determined in a specific form; otherwise, some nonlinear equality constraints will be added into these NLMIs, which is going to make them hard to solve. Even though we determine the structures of some solutions matrices for directed topologies, the results are better than those in [28], which used stronger constraints in solutions as well as a more conservative Lyapunov-Krasovskii functional.

Compared with [28], our results have three improvements. (1) Conservativeness. By optimizing the structure of Lyapunov-Krasovskii functional and adding proper freeweighting matrices, our criterion has less conservativeness. (2) Constraint. Our criterion could permit the case $\mu \geq 1$, even when $\mu$ is unknown (3) Parameters. Both two parameter matrices can be calculated in the design of protocol could calculate directly, and for the second parameter matrix design, our method can give a feasible solution in larger timedelay bounds.

Throughout this paper, the notation represents the symmetric part in a symmetric matrix; $\mathscr{D}>(\geq,<, \leq) 0$ denotes that the matrix $\mathscr{D}$ is positive definite (positive semidefinite, negative definite, negative semidefinite); $\otimes$ denotes the Kronecker product; $\mathbf{0}$ can be an appropriate dimensions zero matrix or vector; for any complex vector $\mathbf{x}$, any real matrix $\mathscr{D}$ and $\lambda \in \mathbb{C}$, we denote $\widehat{\mathbf{x}}=\left[\operatorname{Re}(\mathbf{x})^{T}, \operatorname{Im}(\mathbf{x})^{T}\right]^{T}, \Lambda_{\mathscr{D}}=$

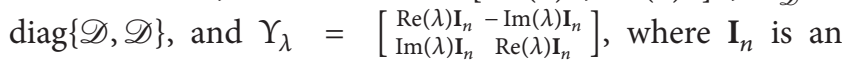
identity matrix with $n \times n$ dimensions.

\section{Preliminaries and Problem Description}

Let $\mathscr{G}=(\mathscr{V}, \mathscr{E}, \mathscr{A})$ be a directed simple graph of order $N(N>1)$, where $\mathscr{V}=\left\{v_{1}, v_{2}, \ldots, v_{N}\right\}$ denotes the nodes set, $\mathscr{E} \subset \mathscr{V} \times \mathscr{V}$ is the edges set, and $\mathscr{A}=\left[\tilde{a}_{i j}\right] \in \mathbb{R}^{N \times N}$ is the weighted adjacency matrix with $\tilde{a}_{i j} \geq 0$, where $\tilde{a}_{i j}>0$ if and only if edge $\left(v_{j}, v_{i}\right) \in \mathscr{E}$. As $\mathscr{G}$ is simple, $\tilde{a}_{i i}=0$ holds for all $i \in\{1,2, \ldots, N\}$. The neighbors of $v_{i}$ is defined as $\mathcal{N}_{i}=\left\{v_{j} \in \mathscr{V} \mid\left(v_{j}, v_{i}\right) \in \mathscr{E}\right\}$. The in-degree of the node $v_{i}$ are defined as $d_{i n}\left(v_{i}\right)=\sum_{v_{j} \in \mathcal{N}_{i}} \widetilde{a}_{i j}$. The degree matrix of $\mathscr{G}$ is a diagonal matrix $\mathscr{D}=\left[d_{i j}\right]$, where $d_{i j}=0(i \neq j), d_{i i}=d_{i n}\left(v_{i}\right)$. Then Laplacian matrix of $\mathscr{G}$ is defined as $\mathscr{L}=\left[l_{i j}\right]=\mathscr{D}-\mathscr{A}$.

Lemma 1 (see [28]). The Laplacian matrix $\mathscr{L}$ of $\mathscr{G}$ satisfies

(1) $\mathscr{L}$ at least has one zero eigenvalue, and $\mathbf{1}_{N}$ is the associated right eigenvector;

(2) if $\mathscr{G}$ has a spanning tree, then 0 is a simple eigenvalue of $\mathscr{L}$, and all the other $N-1$ eigenvalues have positive real parts;

(3) if $\mathscr{G}$ does not have a spanning tree, then $\mathscr{L}$ at least has two zero eigenvalues with the geometric multiplicity being not less than 2.

Lemma 2 (Schur Complement [24]). For a given symmetric matrix $\mathbf{Z}$ with the form $\mathbf{Z}=\mathbf{Z}^{T}=\left[\begin{array}{cc}\mathbf{Z}_{11} & \mathbf{Z}_{12} \\ \star & \mathbf{Z}_{22}\end{array}\right], \mathbf{Z}_{11} \in \mathbb{R}^{r \times r}, \mathbf{Z}_{12} \in$ $\mathbb{R}^{r \times(n-r)}, \mathbf{Z}_{22} \in \mathbb{R}^{(n-r) \times(n-r)}$, then $\mathbf{Z}<0$ if and only if $\mathbf{Z}_{11}<0$, $\mathbf{Z}_{22}-\mathbf{Z}_{12}^{T} \mathbf{Z}_{11}^{-1} \mathbf{Z}_{12}<0$ or $\mathbf{Z}_{22}<0, \mathbf{Z}_{11}-\mathbf{Z}_{12} \mathbf{Z}_{11}^{-1} \mathbf{Z}_{12}^{T}<0$.

In this paper, we consider a group of $N$ agents whose dynamics is described by high-order linear systems in continuous-time domain:

$$
\dot{\mathbf{x}}_{i}(t)=\mathbf{A} \mathbf{x}_{i}(t)+\mathbf{B} \mathbf{u}_{i}(t), \quad i=1,2, \ldots, N,
$$

where $\mathbf{x}_{i}(t)=\left[\begin{array}{llll}x_{i}^{(1)}(t) & x_{i}^{(2)}(t) & \cdots & x_{i}^{(n)}(t)\end{array}\right]^{T}$ is the state of agent $i$ and $\mathbf{u}_{i}(t) \in \mathbb{R}^{p}$ is the consensus protocol, $\mathbf{A} \in \mathbb{R}^{n \times n}$, $\mathbf{B} \in \mathbb{R}^{n \times p}$.

We considered a class of protocols as follows:

$$
\mathbf{u}_{i}(t)=\mathbf{K}_{1} \mathbf{x}_{i}(t)+\mathbf{K}_{2} \sum_{v_{j} \in \mathcal{N}_{i}} \tilde{a}_{i j}\left(\mathbf{x}_{j}(t-\tau(t))-\mathbf{x}_{i}(t-\tau(t))\right),
$$

where $\mathbf{K}_{1}, \mathbf{K}_{2} \in \mathbb{R}^{p \times n}, \tau(t) \in[0, h], \dot{\tau}(t) \in[0, \mu]$.

Let $\mathbf{x}(t)=\left[\begin{array}{llll}\mathbf{x}_{1}^{T}(t) & \mathbf{x}_{2}^{T}(t) & \cdots & \mathbf{x}_{N}^{T}(t)\end{array}\right]^{T}$. With protocol (2), the dynamics of multiagent systems is

$$
\begin{aligned}
\dot{\mathbf{x}}(t)= & \left(\mathbf{I}_{N} \otimes\left(\mathbf{A}+\mathbf{B K}_{1}\right)\right) \mathbf{x}(t) \\
& -\left(\mathscr{L} \otimes \mathbf{B K}_{2}\right) \mathbf{x}(t-\tau(t)), \quad t \in[0,+\infty), \\
\mathbf{x}(t)= & \phi(t), \quad t \in[-h, 0),
\end{aligned}
$$

where $\phi(t)$ is a continuously differentiable initial function.

Definition 3 (consensus). For given $\mathbf{K}_{1}, \mathbf{K}_{2}$, the multiagent systems (3) achieve consensus if and only if for all $i \neq j$, $\lim _{t \rightarrow+\infty}\left\|\mathbf{x}_{i}(t)-\mathbf{x}_{j}(t)\right\|=\mathbf{0}$.

Definition 4 (consensusabilization). Multiagent systems are said to be consensusabilized by $\mathbf{K}_{1}$ and/or $\mathbf{K}_{2}$ if and only if the $\mathbf{K}_{1}$ and/or $\mathbf{K}_{2}$ make the multiagent systems achieve consensus.

\section{Main Results}

In this section, the consensus convergence criterion and protocol parameters design method for high-order linear 
multiagent systems with time-varying delays in directed topologies are provided.

Lemma 5 (see [28]). Dynamics (3) achieves consensus if and only if

$$
\begin{aligned}
\dot{\Delta}_{i}(t)= & \left(\mathbf{A}+\mathbf{B K}_{1}\right) \Delta_{i}(t) \\
& -\lambda_{\mathscr{L}(i+1)} \mathbf{B K}_{2} \Delta_{i}(t-\tau(t)), \quad i=1, \ldots, N-1
\end{aligned}
$$

are asymptotically stable, where $\lambda_{\mathscr{L} j}$ is the jth eigenvalue of the Laplacian Matrix $\mathscr{L}$ corresponding to $\mathscr{G}$, and $0=\operatorname{Re}\left(\lambda_{\mathscr{L} 1}\right) \leq$ $\operatorname{Re}\left(\lambda_{\mathscr{L} 2}\right) \leq \cdots \leq \operatorname{Re}\left(\lambda_{\mathscr{L N}}\right)$.

Remark 6. This necessary and sufficient condition converts the consensus problem in dynamics (3) into a stability problem of dynamics (4). According to Lemma 1, if $\mathscr{G}$ has a spanning tree, there is no $\lambda_{\mathscr{L} i}=0(i=2, \ldots, N)$. Therefore, $\mathbf{K}_{2}$ can make it possible to stabilize systems (4); while if $\mathscr{G}$ does not have a spanning tree, then $\mathscr{L}$ at least has two zero eigenvalues. Without loss of generality, we assume that $\lambda_{\mathscr{L} 2}=$ 0 . Thus, (4) can be rewritten as

$$
\begin{aligned}
\dot{\Delta}_{2}(t) & =\left(\mathbf{A}+\mathbf{B K}_{1}\right) \Delta_{2}(t), \\
\dot{\Delta}_{3}(t) & =\left(\mathbf{A}+\mathbf{B} \mathbf{K}_{1}\right) \Delta_{3}(t)-\lambda_{\mathscr{L} 3} \mathbf{B} \mathbf{K}_{2} \Delta_{3}(t-\tau(t)), \\
& \vdots \\
\dot{\Delta}_{N}(t) & =\left(\mathbf{A}+\mathbf{B} K_{1}\right) \Delta_{N}(t)-\lambda_{\mathscr{L} N} \mathbf{B K}_{2} \Delta_{N}(t-\tau(t)) .
\end{aligned}
$$

If (4) is asymptotically stable, $\mathbf{A}+\mathbf{B} \mathbf{K}_{1}$ should be Hurwitz. In this case, multiagent systems (3) can achieve consensus even if there is no cooperation at all. Therefore, Lemma 5 is adequate to the case with or without spanning trees in communication topology. Therefore, the connectivity assumptions can be neglected.

\subsection{Consensus Convergence Criteria}

Theorem 7. Given the upper bound in communication delay $h$ and its derivative $\mu$, multiagent systems (3) achieve consensus if there exist proper-dimensional $\mathbf{P}_{i}=\mathbf{P}_{i}^{T}>0, \mathbf{Q}_{i}=\mathbf{Q}_{i}^{T} \geq 0$, $\mathbf{R}_{i}=\mathbf{R}_{i}^{T} \geq 0, \mathbf{Z}_{i}=\mathbf{Z}_{i}^{T}>0, \mathbf{X}_{i}=\mathbf{X}_{i}^{T} \geq 0$ and free-weighting matrices

$$
\mathbf{N}_{i}=\left[\begin{array}{l}
\mathbf{N}_{i 1} \\
\mathbf{N}_{i 2} \\
\mathbf{N}_{i 3}
\end{array}\right], \quad \mathbf{S}_{i}=\left[\begin{array}{l}
\mathbf{S}_{i 1} \\
\mathbf{S}_{i 2} \\
\mathbf{S}_{i 3}
\end{array}\right], \quad i=1, \ldots, N-1
$$

such that the following LMIs hold:

$$
\begin{gathered}
\Psi_{i 1}=\left[\begin{array}{cc}
\mathbf{X}_{i} & \mathbf{N}_{i} \\
\star & \mathbf{Z}_{i}
\end{array}\right] \geq 0, \quad \Psi_{i 2}=\left[\begin{array}{cc}
\mathbf{X}_{i} & \mathbf{S}_{i} \\
\star & \mathbf{Z}_{i}
\end{array}\right] \geq 0, \\
\Psi_{i 3}=\left[\begin{array}{cc}
\Phi_{i} & h \widetilde{\mathbf{A}}_{i}^{T} \mathbf{Z}_{i} \\
\star & -h \mathbf{Z}_{i}
\end{array}\right]<0,
\end{gathered}
$$

where $\Phi_{i}=\Phi_{i 1}+\Phi_{i 2}+\Phi_{i 2}^{T}+h \mathbf{X}_{i}$

$$
\begin{aligned}
& \Phi_{i 1} \\
& \begin{array}{c}
=\left[\begin{array}{ccc}
\mathbf{P}_{i} \Lambda_{\mathbf{A}+\mathbf{B K} K_{1}}+\Lambda_{\mathbf{A}+\mathbf{B K} \mathbf{K}_{1}}^{T} \mathbf{P}_{i}+\mathbf{Q}_{i}+\mathbf{R}_{i} & -\Upsilon_{\lambda_{\mathscr{L}(i+1)}} \mathbf{P}_{i} \Lambda_{\mathbf{B K}} & \mathbf{0} \\
\star & -(1-\mu) \mathbf{Q}_{i} & \mathbf{0} \\
\star & \star & -\mathbf{R}_{i}
\end{array}\right] \\
\Phi_{i 2}=\left[\begin{array}{lll}
\mathbf{N}_{i} & -\mathbf{N}_{i}+\mathbf{S}_{i} & -\mathbf{S}_{i}
\end{array}\right] \\
\widetilde{\mathbf{A}}_{i}=\left[\begin{array}{lll}
\Lambda_{\mathbf{A}+\mathbf{B} \mathbf{K}_{1}} & -\Upsilon_{\lambda_{\mathscr{L}(i+1)}} \Lambda_{\mathbf{B K}_{2}} & \mathbf{0}
\end{array}\right]
\end{array}
\end{aligned}
$$

Proof. Since (4) contains complex vectors and matrices, it is required to be transformed into the following form by decompositing the real and imaginary parts:

$$
\begin{aligned}
\dot{\vec{\Delta}}_{i}(t)= & \Lambda_{\mathbf{A}+\mathbf{B K}_{1}} \widehat{\Delta}_{i}(t) \\
& -\Upsilon_{\lambda_{\mathscr{L}(i+1)}} \Lambda_{\mathbf{B K}_{2}} \widehat{\Delta}_{i}(t-\tau(t)), \quad i=1, \ldots, N-1 .
\end{aligned}
$$

Define the following $N-1$ quadratic Lyapunov-Krasovskii functional candidates:

$$
V_{i}(t)=V_{i 1}(t)+V_{i 2}(t)+V_{i 3}(t)+V_{i 4}(t), \quad i=1, \ldots, N-1,
$$

where $V_{i 1}(t)=\widehat{\Delta}_{i}^{T}(t) \mathbf{P}_{i} \widehat{\Delta}_{i}(t), V_{i 2}(t)=\int_{t-\tau(t)}^{t} \widehat{\Delta}_{i}^{T}(s) \mathbf{Q}_{i} \widehat{\Delta}_{i}(s) d s$, $V_{i 3}(t)=\int_{t-h}^{t} \widehat{\Delta}_{i}^{T}(s) \mathbf{R}_{i} \widehat{\Delta}_{i}(s) d s, V_{i 4}(t)=\int_{-h}^{0} \int_{t+\theta}^{t} \dot{\widehat{\Delta}}_{i}^{T}(s) \mathbf{Z}_{i} \dot{\widehat{\Delta}}_{i}(s) d s$ $d \theta$, and $\mathbf{P}_{i}=\mathbf{P}_{i}^{T}>0, \mathbf{Q}_{i}=\mathbf{Q}_{i}^{T} \geq 0, \mathbf{R}_{i}=\mathbf{R}_{i}^{T} \geq 0, \mathbf{Z}_{i}=\mathbf{Z}_{i}^{T}>0$, $\mathbf{X}_{i}=\mathbf{X}_{i}^{T} \geq 0, i=1, \ldots, N-1$.

By Newton-Leibniz formula, for any given free-weighting matrices with proper dimensions

$$
\mathbf{N}_{i}=\left[\begin{array}{l}
\mathbf{N}_{i 1} \\
\mathbf{N}_{i 2} \\
\mathbf{N}_{i 3}
\end{array}\right], \quad \mathbf{S}_{i}=\left[\begin{array}{l}
\mathbf{S}_{i 1} \\
\mathbf{S}_{i 2} \\
\mathbf{S}_{i 3}
\end{array}\right], \quad i=1, \ldots, N-1,
$$

we have

$$
\begin{array}{r}
\widetilde{V}_{i 1}(t)=2 \zeta_{i 1}^{T}(t) \mathbf{N}_{i}\left[\widehat{\Delta}_{i}(t)-\widehat{\Delta}_{i}(t-\tau(t))\right. \\
\left.-\int_{t-\tau(t)}^{t} \dot{\widehat{\Delta}}_{i}(s) d s\right]=\mathbf{0}, \\
\widetilde{V}_{i 2}(t)=2 \zeta_{i 1}^{T}(t) \mathbf{S}_{i}\left[\widehat{\Delta}_{i}(t-\tau(t))-\widehat{\Delta}_{i}(t-h)\right. \\
\left.-\int_{t-\tau(t)}^{t} \dot{\widehat{\Delta}}_{i}(s) d s\right]=\mathbf{0},
\end{array}
$$

where $\zeta_{i 1}(t)=\left[\widehat{\Delta}_{i}^{T}(t), \widehat{\Delta}_{i}^{T}(t-\tau(t)), \widehat{\Delta}_{i}^{T}(t-h)\right]^{T}$.

For any appropriate $\mathbf{X}_{i}=\mathbf{X}_{i}^{T} \geq 0, i=2, \ldots, N$, (13) holds

$$
\begin{aligned}
\widetilde{V}_{i 3}(t)= & h \zeta_{i 1}^{T}(t) \mathbf{X}_{i} \zeta_{i 1}(t)-\int_{t-\tau(t)}^{t} \zeta_{i 1}^{T}(t) \mathbf{X}_{i} \zeta_{i 1}(t) d s \\
& -\int_{t-h}^{t-\tau(t)} \zeta_{i 1}^{T}(t) \mathbf{X}_{i} \zeta_{i 1}(t) d s=\mathbf{0} .
\end{aligned}
$$


Taking the time derivative of $V_{i}(t)$ along the trajectory of (9), it is obtained that

$$
\begin{aligned}
\dot{V}_{i 1}(t)= & \widehat{\Delta}_{i}^{T}(t)\left[\mathbf{P}_{i} \Lambda_{\mathbf{A}+\mathbf{B} \mathbf{K}_{1}}+\Lambda_{\mathbf{A}+\mathbf{B} \mathbf{K}_{1}}^{T} \mathbf{P}_{i}\right] \widehat{\Delta}_{i}(t) \\
& -2 \widehat{\Delta}_{i}^{T}(t) \mathbf{P}_{i} \Upsilon_{\lambda_{\mathscr{L}(i+1)}} \Lambda_{\mathbf{B} \mathbf{K}_{2}} \widehat{\Delta}_{i}(t-\tau(t)), \\
\dot{V}_{i 2}(t)= & \widehat{\Delta}_{i}^{T}(t) \mathbf{Q}_{i} \widehat{\Delta}_{i}(t) \\
& -(1-\dot{\tau}(t)) \widehat{\Delta}_{i}^{T}(t-\tau(t)) \mathbf{Q}_{i} \widehat{\Delta}_{i}(t-\tau(t)), \\
\dot{V}_{i 3}(t)= & \widehat{\Delta}_{i}^{T}(t) \mathbf{R}_{i} \widehat{\Delta}_{i}(t)-\widehat{\Delta}_{i}^{T}(t-h) \mathbf{R}_{i} \widehat{\Delta}_{i}(t-h), \\
\dot{V}_{i 4}(t)= & h\left[\Lambda_{\mathbf{A}+\mathbf{B} \mathbf{K}_{1}} \widehat{\Delta}_{i}(t)-\Upsilon_{\lambda_{\mathscr{L}(i+1)}} \Lambda_{\mathbf{B} \mathbf{K}_{2}} \widehat{\Delta}_{i}(t-\tau(t))\right]^{T} \mathbf{Z}_{i} \\
& \times\left[\Lambda_{\mathbf{A}+\mathbf{B} \mathbf{K}_{1}} \widehat{\Delta}_{i}(t)-\Upsilon_{\lambda_{\mathscr{L}(i+1)}} \Lambda_{\mathbf{B} \mathbf{K}_{2}} \widehat{\Delta}_{i}(t-\tau(t))\right] \\
& -\int_{t-h}^{t} \dot{\hat{\Delta}}_{i}^{T}(s) \mathbf{Z}_{i} \dot{\widehat{\Delta}}_{i}(s) d s .
\end{aligned}
$$

Adding 3 zero items into the derivative, we have

$$
\begin{gathered}
\dot{V}_{i}(t)=\dot{V}_{i 1}(t)+\dot{V}_{i 2}(t)+\dot{V}_{i 3}(t)+\dot{V}_{i 4}(t) \\
+\widetilde{V}_{i 1}(t)+\widetilde{V}_{i 2}(t)+\widetilde{V}_{i 3}(t) .
\end{gathered}
$$

With $\dot{\tau}(t) \leq \mu$, (18) can be zoomed; we can utilize the decomposition of

$$
\begin{aligned}
\int_{t-h}^{t} \dot{\hat{\Delta}}_{i}^{T}(s) \mathbf{Z}_{i} \dot{\hat{\Delta}}_{i}(s) d s= & \int_{t-\tau(t)}^{t} \dot{\hat{\Delta}}_{i}^{T}(s) \mathbf{Z}_{i} \dot{\hat{\Delta}}_{i}(s) d s \\
& +\int_{t-h}^{t-\tau(t)} \dot{\hat{\Delta}}_{i}^{T}(s) \mathbf{Z}_{i} \dot{\widehat{\Delta}}_{i}(s) d s
\end{aligned}
$$

in (17) as a contributor to make the zoomed (18) be quadratic forms:

$$
\begin{aligned}
\dot{V}_{i}(t) \leq & \zeta_{i 1}^{T}(t)\left(\Phi_{i 1}+h \widetilde{\mathbf{A}}_{i}^{T} \mathbf{Z}_{i} \widetilde{\mathbf{A}}_{i}\right) \zeta_{i 1}(t) \\
& -\int_{t-\tau(t)}^{t} \zeta_{i 2}^{T}(t, s)\left[\begin{array}{cc}
\mathbf{X}_{i} & \mathbf{N}_{i} \\
\star & \mathbf{Z}_{i}
\end{array}\right] \zeta_{i 2}(t, s) d s \\
& -\int_{t-h}^{t-\tau(t)} \zeta_{i 2}^{T}(t, s)\left[\begin{array}{cc}
\mathbf{X}_{i} & \mathbf{S}_{i} \\
\star & \mathbf{Z}_{i}
\end{array}\right] \zeta_{i 2}(t, s) d s,
\end{aligned}
$$

where $\zeta_{i 2}(t, s)=\left[\zeta_{i 1}^{T}(t), \dot{\vec{\Delta}}_{i}^{T}(s)\right]^{T}$. For formula $(20)$, if $\Phi_{i 1}+$ $h \widetilde{\mathbf{A}}_{i}^{T} \mathbf{Z}_{i} \widetilde{\mathbf{A}}_{i}<0$ and

$$
\Psi_{i 1}=\left[\begin{array}{cc}
\mathbf{X}_{i} & \mathbf{N}_{i} \\
\star & \mathbf{Z}_{i}
\end{array}\right] \geq 0, \quad \Psi_{i 2}=\left[\begin{array}{cc}
\mathbf{X}_{i} & \mathbf{S}_{i} \\
\star & \mathbf{Z}_{i}
\end{array}\right] \geq 0,
$$

then $\dot{V}_{i}(t)<-\varepsilon\left\|\Delta_{i}(t)\right\|^{2}$ holds for any $\varepsilon>0, i=1, \ldots, N-1$.

By Lemma 2, $\Phi_{i 1}+h \widetilde{\mathbf{A}}_{i}^{T} \mathbf{Z}_{i} \widetilde{\mathbf{A}}_{i}<0$ equals to $\Psi_{i 3}<0$. Thus, if (7), holds then (9) is asymptotically stable and multiagent systems (3) achieve consensus.

Remark 8. Theorem 7 has only one zoom in the derivative of Lyapunov-Krasovskii functional which is necessary, but
Theorem 2 in [28] has three zooms, which make the criterion have more conservativeness. Numerical examples and Simulations, in Section 4.1, will validate that our Theorem 7 has less conservativeness.

Remark 9. Setting $\mathbf{Q}_{i}=\mathbf{0}$ for all $i \in\{1,2, \ldots, N-1\}$, we can get the delay-dependent and rate-independent consensus convergence criterion; while Theorem 2 in [28] requires that the upper bounds of $\mu$ are limited by $\mu<1$.

3.2. Protocol Parameters Design Algorithm in Undirected Communication Topology. In this section, the algorithms in designing protocol parameter matrices in (2) under undirected communication topologies are investigated (including single parameter matrix design and both parameter matrices design).

Theorem 10 (double protocol parameter matrices design for undirected communication topology). Assume that the topology $\mathscr{G}$ is an undirected graph. Given the upper bound in communication delay $h$ and its derivative $\mu$, if there exist proper-dimensional $\overline{\mathbf{L}}=\overline{\mathbf{L}}^{T}>0, \overline{\mathbf{W}}_{i}=\overline{\mathbf{W}}_{i}^{T} \geq 0, \overline{\mathbf{U}}_{i}=\overline{\mathbf{U}}_{i}^{T} \geq 0$, $\overline{\mathbf{G}}_{i}=\overline{\mathbf{G}}_{i}^{T} \geq 0$

$$
\overline{\mathbf{Y}}_{i}=\overline{\mathbf{Y}}_{i}^{T}=\left[\begin{array}{ccc}
\overline{\mathbf{Y}}_{11}^{(i)} & \overline{\mathbf{Y}}_{12}^{(i)} & \overline{\mathbf{Y}}_{13}^{(i)} \\
\star & \overline{\mathbf{Y}}_{22}^{(i)} & \overline{\mathbf{Y}}_{23}^{(i)} \\
\star & \star & \overline{\mathbf{Y}}_{33}^{(i)}
\end{array}\right] \geq 0
$$

and free-weighting matrices $\overline{\mathbf{V}}_{1}, \overline{\mathbf{V}}_{2}$

$$
\overline{\mathbf{M}}_{i}=\left[\begin{array}{l}
\overline{\mathbf{M}}_{i 1} \\
\overline{\mathbf{M}}_{i 2} \\
\overline{\mathbf{M}}_{i 3}
\end{array}\right], \quad \overline{\mathbf{T}}_{i}=\left[\begin{array}{l}
\overline{\mathbf{T}}_{i 1} \\
\overline{\mathbf{T}}_{i 2} \\
\overline{\mathbf{T}}_{i 3}
\end{array}\right], \quad i=1, \ldots, N-1
$$

such that the following NLMIs hold:

$$
\bar{\Psi}_{i 1}=\left[\begin{array}{cc}
\overline{\mathbf{Y}}_{i} & \overline{\mathbf{M}}_{i} \\
\star & \overline{\mathbf{L}} \overline{\mathbf{G}}_{i}^{-1} \overline{\mathbf{L}}
\end{array}\right] \geq 0, \quad \bar{\Psi}_{i 2}=\left[\begin{array}{cc}
\overline{\mathbf{Y}}_{i} & \overline{\mathbf{T}}_{i} \\
\star & \overline{\mathbf{L}} \overline{\mathbf{G}}_{i}^{-1} \overline{\mathbf{L}}
\end{array}\right] \geq 0,
$$

$$
\bar{\Psi}_{i 3}=\left[\begin{array}{cccc}
\bar{\Pi}_{11}^{(i)} & \bar{\Pi}_{12}^{(i)} & \bar{\Pi}_{13}^{(i)} & h \overline{\mathbf{L}} \mathbf{A}^{T}+h \overline{\mathbf{V}}_{1}^{T} \mathbf{B}^{T} \\
\star & \bar{\Pi}_{22}^{(i)} & \bar{\Pi}_{23}^{(i)} & -h \lambda_{\mathscr{L}(i+1)} \overline{\mathbf{V}}_{2}^{T} \mathbf{B}^{T} \\
\star & \star & \Pi_{33}^{(i)} & \mathbf{0} \\
\star & \star & \star & -h \overline{\mathbf{G}}_{i}
\end{array}\right]<0,
$$


$i=1, \ldots, N-1$, where

$$
\begin{aligned}
\bar{\Pi}_{11}^{(i)}= & \mathbf{A} \overline{\mathbf{L}}+\overline{\mathbf{L}}^{T}+\mathbf{B} \overline{\mathbf{V}}_{1}+\overline{\mathbf{V}}_{1}^{T} \mathbf{B}^{T}+\overline{\mathbf{W}}_{i} \\
& +\overline{\mathbf{U}}_{i}+\overline{\mathbf{M}}_{i 1}+\overline{\mathbf{M}}_{i 1}^{T}+h \overline{\mathbf{Y}}_{11}^{(i)}, \\
\bar{\Pi}_{12}^{(i)}= & -\lambda_{\mathscr{L}(i+1)} \mathbf{B} \overline{\mathbf{V}}_{2}-\overline{\mathbf{M}}_{i 1}+\overline{\mathbf{T}}_{i 1}+\overline{\mathbf{M}}_{i 2}^{T}+h \overline{\mathbf{Y}}_{12}^{(i)}, \\
\bar{\Pi}_{13}^{(i)}= & -\overline{\mathbf{T}}_{i 1}+\overline{\mathbf{M}}_{i 3}^{T}+h \overline{\mathbf{Y}}_{13}^{(i)}, \\
\bar{\Pi}_{22}^{(i)}= & -(1-\mu) \overline{\mathbf{W}}_{i}-\overline{\mathbf{M}}_{i 2}-\overline{\mathbf{M}}_{i 2}^{T}+\overline{\mathbf{T}}_{i 2}+\overline{\mathbf{T}}_{i 2}^{T}+h \overline{\mathbf{Y}}_{22}^{(i)}, \\
\bar{\Pi}_{23}^{(i)}= & -\overline{\mathbf{T}}_{i 2}-\overline{\mathbf{M}}_{i 3}^{T}+\overline{\mathbf{T}}_{i 3}^{T}+h \overline{\mathbf{Y}}_{23}^{(i)}, \\
\bar{\Pi}_{33}^{(i)}= & -\overline{\mathbf{U}}_{i}-\overline{\mathbf{T}}_{i 3}-\overline{\mathbf{T}}_{i 3}^{T}+h \overline{\mathbf{Y}}_{33}^{(i)},
\end{aligned}
$$

then multiagent systems (1) can be consensusabilized by proto$\operatorname{col}(2)$, and the parameters are from $\mathbf{K}_{1}=\overline{\mathbf{V}}_{1} \overline{\mathbf{L}}^{-1}, \mathbf{K}_{2}=\overline{\mathbf{V}}_{2} \overline{\mathbf{L}}^{-1}$.

Proof. If multiagent systems (3) achieve consensus, then according to Theorem 7, inequalities (7) hold. Note that $\mathscr{L}$ is symmetrical due to a undirected $\mathscr{G}$; we can halve the dimension of $\Psi_{i 1}$ and $\Psi_{i 2}$ in (7). Further, $\Psi_{i 3}$ can be rewritten as follows:

$$
\Psi_{i 3}=\left[\begin{array}{cc}
\Phi_{i} & h \widetilde{\mathbf{A}}_{i}^{T} \mathbf{Z}_{i} \\
\star & -h \mathbf{Z}_{i}
\end{array}\right]<0,
$$

where $\Phi_{i}=\Phi_{i 1}+\Phi_{i 2}+\Phi_{i 2}^{T}+h \mathbf{X}_{i}$

$$
\begin{aligned}
& \Phi_{i 1} \\
& =\left[\begin{array}{ccc}
\mathbf{P}_{i}\left(\mathbf{A}+\mathbf{B K}_{1}\right)+\left(\mathbf{A}+\mathbf{B K}_{1}\right)^{T} \mathbf{P}_{i}+\mathbf{Q}_{i}+\mathbf{R}_{i} & -\lambda_{\mathscr{L}(i+1)} \mathbf{P}_{i} \mathbf{B} \mathbf{K}_{2} & \mathbf{0} \\
\star & -(1-\mu) \mathbf{Q}_{i} & \mathbf{0} \\
\star & \star & -\mathbf{R}_{i}
\end{array}\right], \\
& \Phi_{i 2}=\left[\begin{array}{lll}
\mathbf{N}_{i} & -\mathbf{N}_{i}+\mathbf{S}_{i}-\mathbf{S}_{i}
\end{array}\right], \\
& \widetilde{\mathbf{A}}_{i}=\left[\begin{array}{lll}
\mathbf{A}+\mathbf{B K}_{1} & -\lambda_{\mathscr{L}(i+1)} \mathbf{B K}_{2} & \mathbf{0}
\end{array}\right] .
\end{aligned}
$$

Then employing the congruent transformation with $\operatorname{diag}\left\{\mathbf{P}_{i}^{-1}, \mathbf{P}_{i}^{-1}, \mathbf{P}_{i}^{-1}, \mathbf{Z}_{i}^{-1}\right\}$ for $\Psi_{i 3}$ in (27), we can derive

$$
\operatorname{diag}\left\{\mathbf{P}_{i}^{-1}, \mathbf{P}_{i}^{-1}, \mathbf{P}_{i}^{-1}, \mathbf{Z}_{i}^{-1}\right\}^{T} \Psi_{i 3} \operatorname{diag}\left\{\mathbf{P}_{i}^{-1}, \mathbf{P}_{i}^{-1}, \mathbf{P}_{i}^{-1}, \mathbf{Z}_{i}^{-1}\right\}<0,
$$

where $i=1, \ldots, N-1$. Set $\mathbf{P}_{1}^{-1}=\mathbf{P}_{2}^{-1}=\cdots=\mathbf{P}_{N-1}^{-1}=\overline{\mathbf{L}}$, and let $\overline{\mathbf{W}}_{i}=\overline{\mathbf{L}} \mathbf{Q}_{i} \overline{\mathbf{L}}, \overline{\mathbf{U}}_{i}=\overline{\mathbf{L}} \mathbf{R}_{i} \overline{\mathbf{L}}, \overline{\mathbf{G}}_{i}=\mathbf{Z}_{i}^{-1}$ and

$$
\begin{gathered}
\overline{\mathbf{Y}}_{i}=\overline{\mathbf{L}} \mathbf{X}_{i} \overline{\mathbf{L}}=\left[\begin{array}{ccc}
\overline{\mathbf{Y}}_{11}^{(i)} & \overline{\mathbf{Y}}_{12}^{(i)} & \overline{\mathbf{Y}}_{13}^{(i)} \\
\star & \overline{\mathbf{Y}}_{22}^{(i)} & \overline{\mathbf{Y}}_{23}^{(i)} \\
\star & \star & \overline{\mathbf{Y}}_{33}^{(i)}
\end{array}\right] \geq 0, \\
\overline{\mathbf{M}}_{i}=\operatorname{diag}\{\overline{\mathbf{L}}, \overline{\mathbf{L}}, \overline{\mathbf{L}}\} \mathbf{N}_{i} \overline{\mathbf{L}}=\left[\begin{array}{l}
\overline{\mathbf{M}}_{i 1} \\
\overline{\mathbf{M}}_{i 2} \\
\overline{\mathbf{M}}_{i 3}
\end{array}\right], \\
\overline{\mathbf{T}}_{i}=\operatorname{diag}\{\overline{\mathbf{L}}, \overline{\mathbf{L}}, \overline{\mathbf{L}}\} \mathbf{S}_{i} \overline{\mathbf{L}}=\left[\begin{array}{l}
\overline{\mathbf{T}}_{i 1} \\
\overline{\mathbf{T}}_{i 2} \\
\overline{\mathbf{T}}_{i 3}
\end{array}\right],
\end{gathered}
$$

where $i=1, \ldots, N-1$ (note that the $\mathbf{P}_{i}, \mathbf{Q}_{i}, \mathbf{R}_{i}, \mathbf{Z}_{i}, \mathbf{X}_{i}, \mathbf{N}_{i}, \mathbf{S}_{i}$ are half dimensional compared to those in Theorem 7). Subsequently, with $\mathbf{K}_{1} \overline{\mathbf{L}}=\overline{\mathbf{V}}_{1}$ and $\mathbf{K}_{2} \overline{\mathbf{L}}=\overline{\mathbf{V}}_{2}$, formula (29) can be rewritten as (25). Similarly, by using congruent transformation with $\operatorname{diag}\{\overline{\mathbf{L}}, \overline{\mathbf{L}}, \overline{\mathbf{L}}\}$ in dimension-halved $\Psi_{i 1}$ and $\Psi_{i 2}$, respectively, inequalities (24) can be derived. Note that (24) and (25) are equivalent to (7); the protocol parameters are from $\mathbf{K}_{1}=\overline{\mathbf{V}}_{1} \overline{\mathbf{L}}^{-1}, \mathbf{K}_{2}=\overline{\mathbf{V}}_{2} \overline{\mathbf{L}}^{-1}$.

Remark 11. To avoid divergence of the multiagent systems, sometimes we need to design $\mathbf{K}_{1}, \mathbf{K}_{2}$ at the same time, even though it is proven in [28] that the $\mathbf{K}_{1}$ is responsible for the state trajectories of those multiagent systems which achieve consensus. NLMIs (24) and (25) can be solved by using Cone Complementarity Linearization (CCL) method (see $[30,31]$ ).

Remark 12. In order to design the $\mathbf{K}_{2}$ only, we can set $\mathbf{K}_{1}$ equal to a certain constant matrix in Theorem 10. Accordingly, our Theorem 10 can be compared with Theorem 3 in [28].

\subsection{Protocol Parameters Design Algorithm in Directed Com-} munication Topology. In this section, the algorithms in designing protocol parameter matrices in (2) under directed communication topologies are proposed.

Note that if the topology is directed, the structure of freeweighting matrices $\overline{\mathbf{V}}_{1}, \overline{\mathbf{V}}_{2}$ is limited by $\Lambda_{\mathbf{K}_{1}}=\overline{\mathbf{V}}_{1} \overline{\mathbf{L}}^{-1}$ and $\Lambda_{\mathbf{K}_{2}}=\overline{\mathbf{V}}_{2} \overline{\mathbf{L}}^{-1}$ (actually, the limitation caused by diagonal structures of $\Lambda_{\mathbf{K}_{1}}, \Lambda_{\mathbf{K}_{2}}$ ). That means even though we calculate the free-weighting matrices $\overline{\mathbf{V}}_{1}, \overline{\mathbf{V}}_{2}$ by solving NLMIs, we could not always acquire a diagonal structured $\Lambda_{\mathbf{K}_{1}}, \Lambda_{\mathbf{K}_{2}}$. More importantly, both the diagonal blocks in $\mathbf{K}_{1}$ (as well as in $\mathbf{K}_{2}$ ) are required to be equal. This will add nonlinear equality constraints in NLMIs, which are hard to solve.

Therefore, we determine the structures of $\overline{\mathbf{V}}_{1}, \overline{\mathbf{V}}_{2}$ as well as $\overline{\mathbf{L}}$ in advance. Theorem 13 gives the corresponding NLMIs.

Theorem 13 (double protocol parameter matrices design for directed communication topology). Assume the topology $\mathscr{G}$ is a directed graph. Given the upper bound in communication 
delay $h$ and its derivative $\mu$, if there exist proper-dimensional $\overline{\mathbf{L}}=\overline{\mathbf{L}}^{T}>0, \overline{\mathbf{W}}_{i}=\overline{\mathbf{W}}_{i}^{T} \geq 0, \overline{\mathbf{U}}_{i}=\overline{\mathbf{U}}_{i}^{T} \geq 0, \overline{\mathbf{G}}_{i}=\overline{\mathbf{G}}_{i}^{T} \geq 0$

$$
\overline{\mathbf{Y}}_{i}=\overline{\mathbf{Y}}_{i}^{T}=\left[\begin{array}{ccc}
\overline{\mathbf{Y}}_{11}^{(i)} & \overline{\mathbf{Y}}_{12}^{(i)} & \overline{\mathbf{Y}}_{13}^{(i)} \\
\star & \overline{\mathbf{Y}}_{22}^{(i)} & \overline{\mathbf{Y}}_{23}^{(i)} \\
\star & \star & \overline{\mathbf{Y}}_{33}^{(i)}
\end{array}\right] \geq 0
$$

and free-weighting matrices $\overline{\mathbf{V}}_{1}, \overline{\mathbf{V}}_{2}$,

$$
\overline{\mathbf{M}}_{i}=\left[\begin{array}{l}
\overline{\mathbf{M}}_{i 1} \\
\overline{\mathbf{M}}_{i 2} \\
\overline{\mathbf{M}}_{i 3}
\end{array}\right], \quad \overline{\mathbf{T}}_{i}=\left[\begin{array}{c}
\overline{\mathbf{T}}_{i 1} \\
\overline{\mathbf{T}}_{i 2} \\
\overline{\mathbf{T}}_{i 3}
\end{array}\right], \quad i=1, \ldots, N-1
$$

such that the following NLMIs hold:

$$
\begin{aligned}
& \bar{\Psi}_{i 1}=\left[\begin{array}{cc}
\overline{\mathbf{Y}}_{i} & \overline{\mathbf{M}}_{i} \\
\star & \overline{\mathbf{L G}}_{i}^{-1} \overline{\mathbf{L}}
\end{array}\right] \geq 0, \quad \bar{\Psi}_{i 2}=\left[\begin{array}{cc}
\overline{\mathbf{Y}}_{i} & \overline{\mathbf{T}}_{i} \\
\star & \overline{\mathbf{L}} \overline{\mathbf{G}}_{i}^{-1} \overline{\mathbf{L}}
\end{array}\right] \geq 0, \\
& \bar{\Psi}_{i 3}=\left[\begin{array}{cccc}
\bar{\Pi}_{11}^{(i)} & \bar{\Pi}_{12}^{(i)} & \bar{\Pi}_{13}^{(i)} & h\left(\Lambda_{\mathbf{A}} \overline{\mathbf{L}}\right)^{T}+h\left(\Lambda_{\mathbf{B}} \overline{\mathbf{V}}_{1}\right)^{T} \\
\star & \bar{\Pi}_{22}^{(i)} & \bar{\Pi}_{23}^{(i)} & -h\left(\Upsilon_{\lambda_{\mathscr{L}(i+1)}} \Lambda_{\mathbf{B}} \overline{\mathbf{V}}_{2}\right)^{T} \\
\star & \star & \bar{\Pi}_{33}^{(i)} & \mathbf{0} \\
\star & \star & \star & -h \overline{\mathbf{G}}_{i}
\end{array}\right]<0
\end{aligned}
$$

$i=1, \ldots, N-1$, where

$$
\begin{aligned}
\bar{\Pi}_{11}^{(i)}= & \Lambda_{\mathbf{A}} \overline{\mathbf{L}}+\left(\Lambda_{\mathbf{A}} \overline{\mathbf{L}}\right)^{T}+\Lambda_{\mathbf{B}} \overline{\mathbf{V}}_{1}+\left(\Lambda_{\mathbf{B}} \overline{\mathbf{V}}_{1}\right)^{T}+\overline{\mathbf{W}}_{i} \\
& +\overline{\mathbf{U}}_{i}+\overline{\mathbf{M}}_{i 1}+\overline{\mathbf{M}}_{i 1}^{T}+h \overline{\mathbf{Y}}_{11}^{(i)}, \\
\bar{\Pi}_{12}^{(i)}= & -\Upsilon_{\lambda_{\mathscr{S}(i+1)}} \Lambda_{\mathbf{B}} \overline{\mathbf{V}}_{2}-\overline{\mathbf{M}}_{i 1}+\overline{\mathbf{T}}_{i 1}+\overline{\mathbf{M}}_{i 2}^{T}+h \overline{\mathbf{Y}}_{12}^{(i)}, \\
\bar{\Pi}_{13}^{(i)}= & -\overline{\mathbf{T}}_{i 1}+\overline{\mathbf{M}}_{i 3}^{T}+h \overline{\mathbf{Y}}_{13}^{(i)}, \\
\bar{\Pi}_{22}^{(i)}= & -(1-\mu) \overline{\mathbf{W}}_{i}-\overline{\mathbf{M}}_{i 2}-\overline{\mathbf{M}}_{i 2}^{T}+\overline{\mathbf{T}}_{i 2}+\overline{\mathbf{T}}_{i 2}^{T}+h \overline{\mathbf{Y}}_{22}^{(i)}, \\
\bar{\Pi}_{23}^{(i)}= & -\overline{\mathbf{T}}_{i 2}-\overline{\mathbf{M}}_{i 3}^{T}+\overline{\mathbf{T}}_{i 3}^{T}+h \overline{\mathbf{Y}}_{23}^{(i)}, \\
\bar{\Pi}_{33}^{(i)}= & -\overline{\mathbf{U}}_{i}-\overline{\mathbf{T}}_{i 3}-\overline{\mathbf{T}}_{i 3}^{T}+h \overline{\mathbf{Y}}_{33}^{(i)}
\end{aligned}
$$

and the structures of $\overline{\mathbf{L}}, \overline{\mathbf{V}}_{1}, \overline{\mathbf{V}}_{2}$ are

$$
\begin{gathered}
\overline{\mathbf{L}}=\left[\begin{array}{cc}
\overline{\mathbf{L}}_{11} & \overline{\mathbf{L}}_{12} \\
\star & \overline{\mathbf{L}}_{22}
\end{array}\right]>0, \quad \overline{\mathbf{V}}_{1}=\left[\begin{array}{cc}
\overline{\mathbf{V}}_{11}^{(1)} & \overline{\mathbf{V}}_{12}^{(1)} \\
\overline{\mathbf{V}}_{21}^{(1)} & \overline{\mathbf{V}}_{22}^{(1)}
\end{array}\right], \\
\overline{\mathbf{V}}_{2}=\left[\begin{array}{cc}
\overline{\mathbf{V}}_{11}^{(2)} & \overline{\mathbf{V}}_{12}^{(2)} \\
\overline{\mathbf{V}}_{21}^{(2)} & \overline{\mathbf{V}}_{22}^{(2)}
\end{array}\right],
\end{gathered}
$$

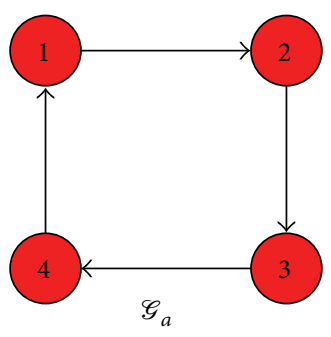

(a)

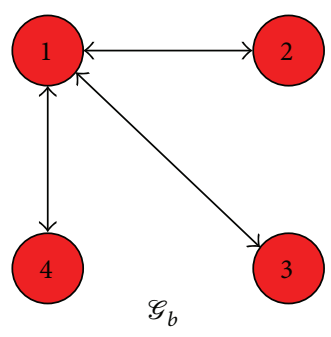

(b)
Figure 1: Two directed graphs.

where $\overline{\mathbf{L}}_{11}=\overline{\mathbf{L}}_{22}, \overline{\mathbf{L}}_{12}=\mathbf{0}, \overline{\mathbf{V}}_{11}^{(i)}=\overline{\mathbf{V}}_{22}^{(i)}=\mathbf{0}, \overline{\mathbf{V}}_{12}^{(i)}=\overline{\mathbf{V}}_{21}^{(i)}=\mathbf{0}$, $i=1,2$, then multiagent systems (1) can be consensusablized by protocol (2), and the parameters are from $\mathbf{K}_{1}=\overline{\mathbf{V}}_{11}^{(1)} \overline{\mathbf{L}}_{11}^{-1}$, $\mathbf{K}_{2}=\overline{\mathbf{V}}_{11}^{(2)} \overline{\mathbf{L}}_{11}^{-1}$.

Proof. The process of proof is similar to that in Theorem 10. The differences are as follows: (1) the dimension of matrices is doubled compared to those in Theorem 10; (2) the structures of $\overline{\mathbf{L}}, \overline{\mathbf{V}}_{1}, \overline{\mathbf{V}}_{2}$ are restricted by (35) for the sake of eliminating the nonlinear equality constraints.

Remark 14. Similar to Remark 11, we can set $\mathbf{K}_{1}$ equal to a certain constant matrix in Theorem 13 so that the $\mathbf{K}_{2}$ can be designed under the condition that $\mathbf{K}_{1}$ has already been chosen.

\section{Numerical Examples and Simulations}

In order to validate the theoretical results proposed in this paper, numerical examples and simulations are involved to illustrate the effectiveness. Figure 1 reveals some topologies in multiagent systems. For simplicity, assume that their adjacency matrices are limited to 0,1 matrices.

4.1. Comparisons on Consensus Convergence Criteria. Firstly, we will compare the conservativeness of the criteria with [28]. Additionally, the applicability for unknown $\mu$ in our criterion will be illustrated.

Consider multiagent systems (3) with the following parameters:

$$
\begin{array}{rlrl}
\mathbf{A} & =\left[\begin{array}{ccc}
0 & 1 & 0 \\
0 & 0 & 1 \\
-3 & -1 & -2
\end{array}\right], & \mathbf{B}=\left[\begin{array}{ll}
0 & 0 \\
0 & 1 \\
1 & 0
\end{array}\right], \\
\mathbf{K}_{1}=\left[\begin{array}{ccc}
3 & 1 & 2 \\
-1 & -1 & -1
\end{array}\right], & \mathbf{K}_{2}=\left[\begin{array}{lll}
0 & 0 & 1 \\
5 & 4 & 1
\end{array}\right] .
\end{array}
$$

The initial function is

$$
\phi(t) \equiv\left[\phi_{1}^{T}(t), \phi_{2}^{T}(t), \phi_{3}^{T}(t), \phi_{4}^{T}(t)\right]^{T}, \quad t \in[-h, 0),
$$




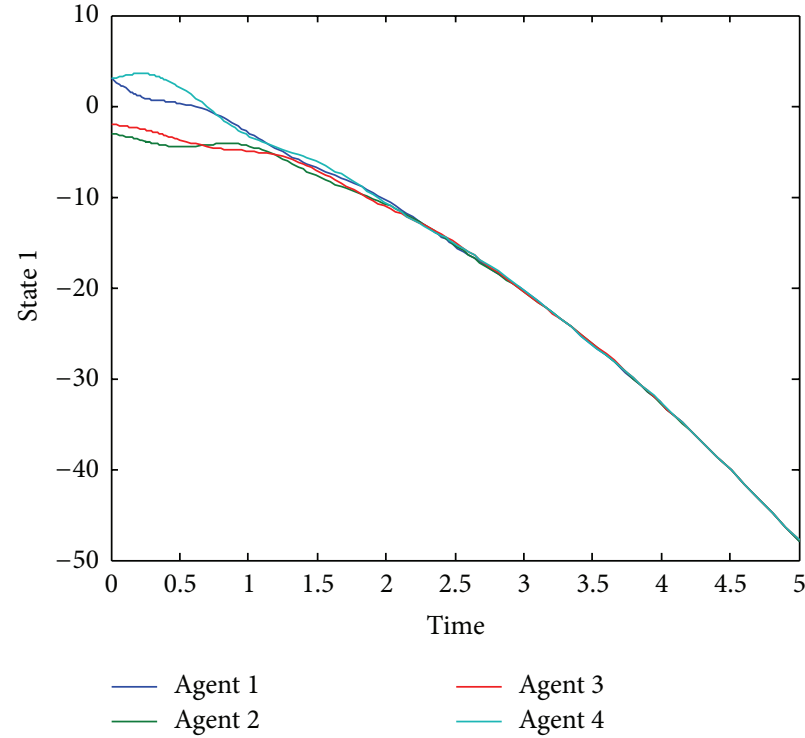

(a)

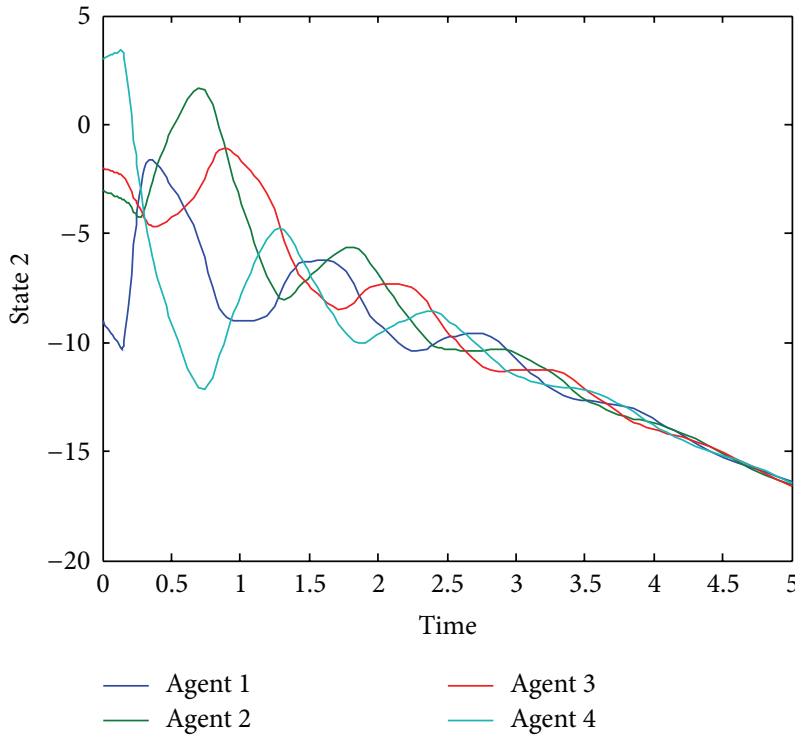

(b)

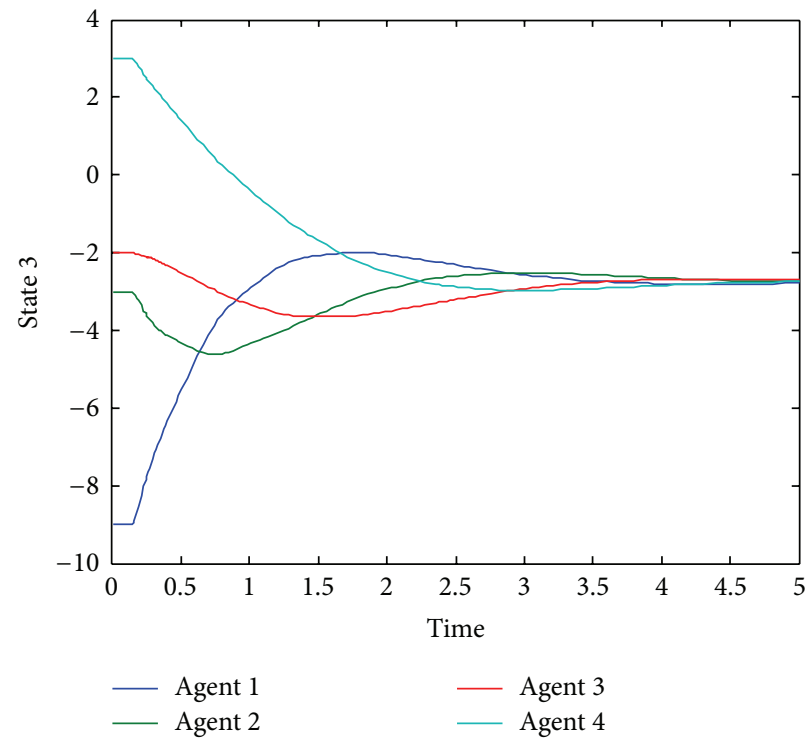

(c)

Figure 2: State trajectories of 3rd-order multiagent systems in $\mathscr{G}_{a}$ with dynamics (36), protocol parameters (37), and communication delay $\tau(t)=0.149 / 2+(0.149 / 2) \sin ((2 \times 0.9 / 0.149) t)$.

where $h$ is the upper bound on communication delay $\tau(t)$ (see formula (3)) and

$$
\begin{aligned}
\phi_{1}(t) & \equiv\left[\begin{array}{c}
3 \\
-9 \\
-9
\end{array}\right], & \phi_{2}(t) \equiv\left[\begin{array}{l}
-3 \\
-3 \\
-3
\end{array}\right], \\
\phi_{3}(t) & \equiv\left[\begin{array}{l}
-2 \\
-2 \\
-2
\end{array}\right], & \phi_{4}(t) \equiv\left[\begin{array}{l}
3 \\
3 \\
3
\end{array}\right] .
\end{aligned}
$$

Results of comparisons are shown in Table 1 .

Remark 15. The comparison results illustrate that allowable upper bound on communication delays given by Theorem 7
TABLE 1: Allowable upper bound on communication delay for thirdorder linear multiagent systems with topology $\mathscr{G}_{a}, \mathscr{G}_{b}$.

\begin{tabular}{lcccc}
\hline & $\mu=0$ & $\mu=0.5$ & $\mu=0.9$ & Unknown $\mu$ \\
\hline [28, Theorem 2] $\mathscr{G}_{a}$ & 0.097 & 0.097 & 0.097 & - \\
Theorem 7 $\mathscr{G}_{a}$ & 0.151 & 0.149 & 0.149 & 0.149 \\
[28, Theorem 2] $\mathscr{G}_{b}$ & 0.074 & 0.067 & 0.063 & - \\
Theorem 7 $\mathscr{G}_{b}$ & 0.084 & 0.080 & 0.080 & 0.080 \\
\hline
\end{tabular}

is larger than those in [28] (to be more precise, ranging from $13.51 \%$ to $55.67 \%$ ); the simulation results in Figure 2 validate the effectiveness. More importantly, our criterion has more 


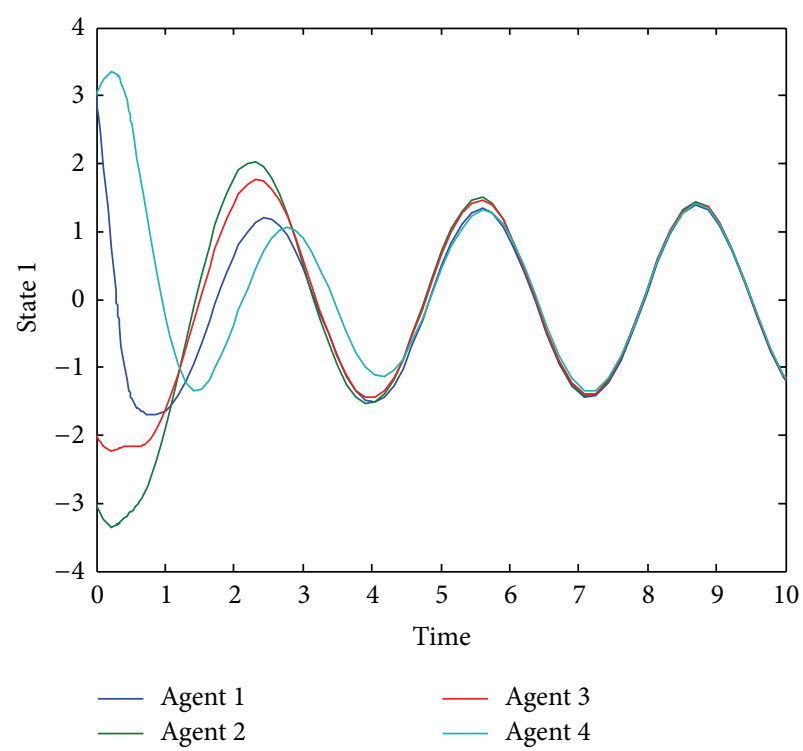

(a)

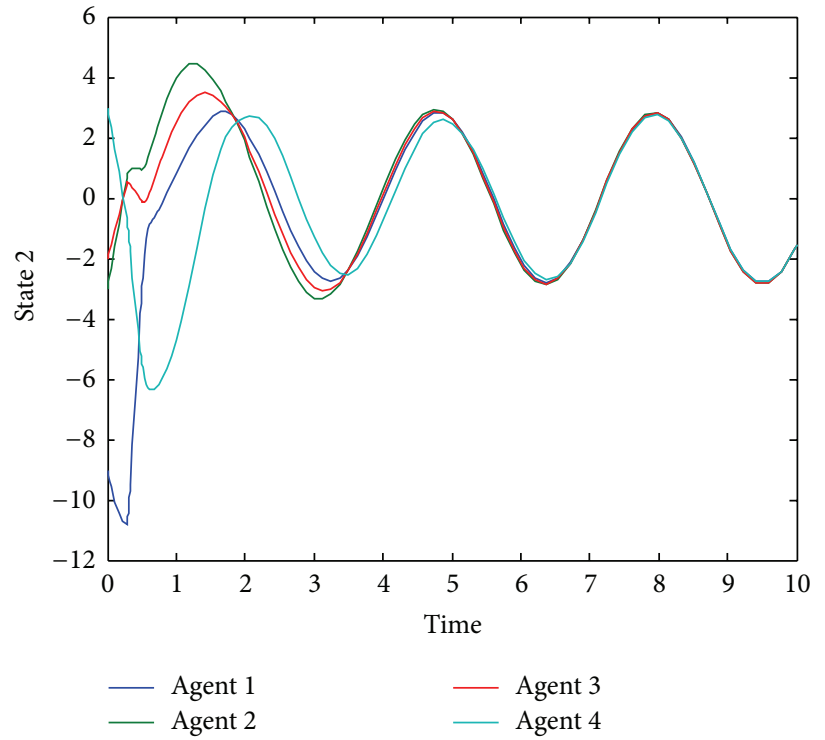

(b)

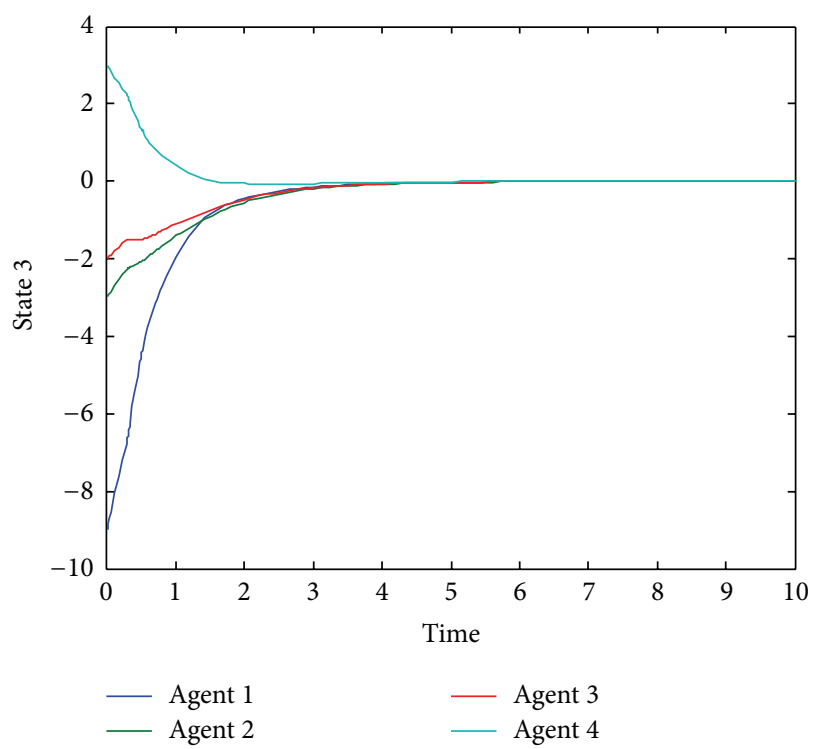

(c)

FIGURE 3: State trajectories of 3rd-order multiagent systems in $\mathscr{G}_{b}$ with protocol parameters $(40)$ and $(42)$ and communication delay $\tau(t)=$ $0.295 / 2+(0.295 / 2) \sin ((2 \times 0.9 / 0.295) t)$.

advantages when $\mu$ becomes larger. Especially, unknown $\mu$ is able to judge by our criterion.

For Theorem 2 in [28], the number of LyapunovKrasovskii functional candidates is reduced to 2 with the aim of saving computing time, even though this would lead to some conservativeness. Similarly, in the current paper, we could also reduce the candidates' number to 2 to get a higher computation efficiency. But still, for a more persuaded comparison, we select the all $(N-1)$ Lyapunov-Krasovskii functional candidates.
4.2. Validations for Protocol Design Algorithm. Firstly, our Theorem 10 is proposed to design the parameter matrices in protocol (2) under undirected communication topologies, including (1) calculating $\mathbf{K}_{2}$ with a known $\mathbf{K}_{1}$, (the comparisons with Theorem 3 in [28] are made in Table 2); (2) determining both of the parameter matrices $\mathbf{K}_{1}$ and $\mathbf{K}_{2}$ (while in [28], $\mathbf{K}_{1}$ could not be calculated automatically by its theorems) and results are shown in Table 3.

Secondly, for directed communication topologies, the parameter matrices of protocol (2) are determined by our Theorem 13. Similarly, both cases (designing $\mathbf{K}_{2}$ only or 


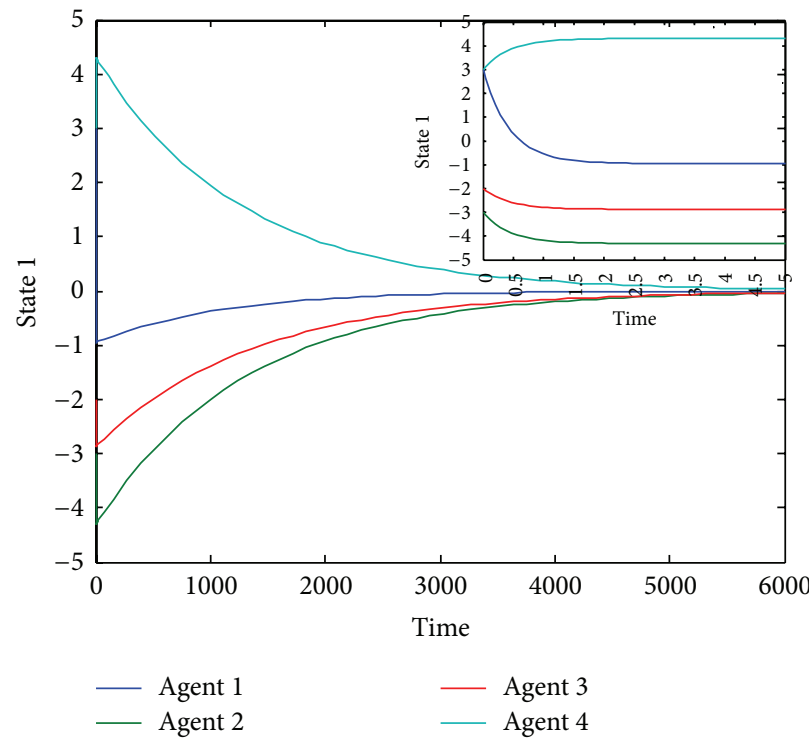

(a)

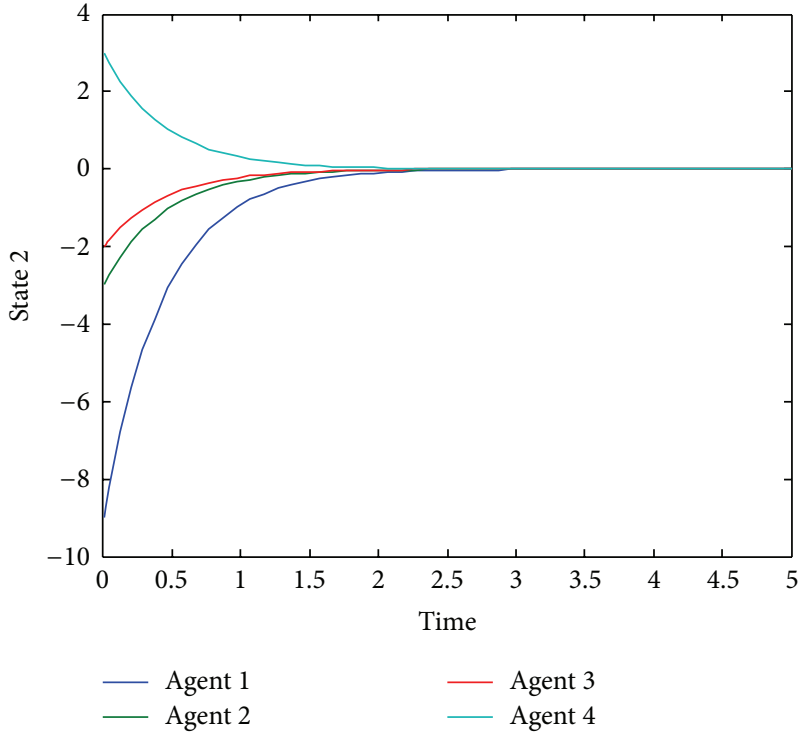

(b)

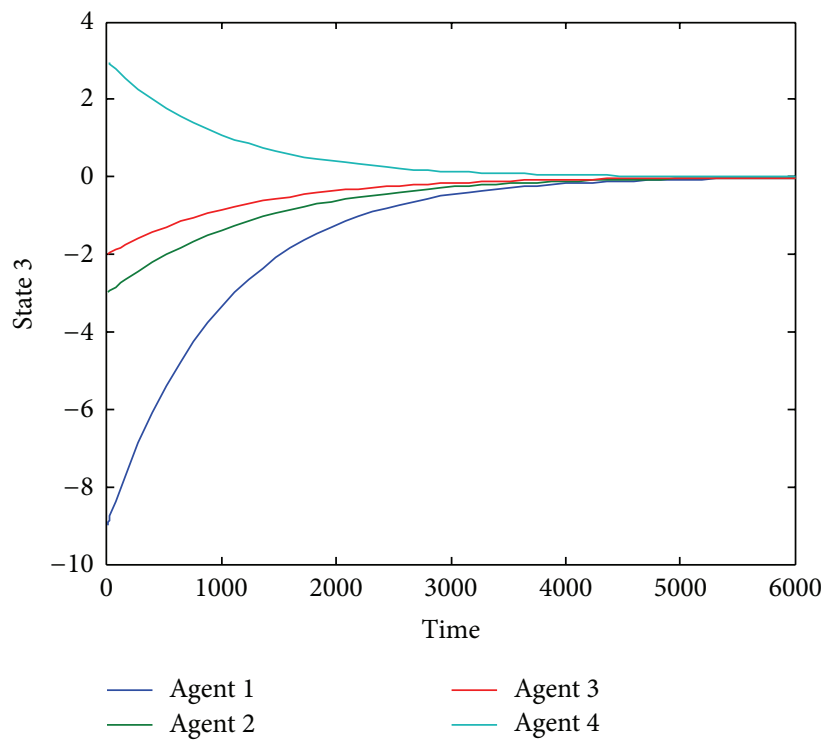

(c)

FIgURE 4: State trajectories of 3rd-order multiagent systems in $\mathscr{G}_{a}$ with protocol parameters design $(h=0.1, \mu=0.9)$.

calculating $\mathbf{K}_{1}, \mathbf{K}_{2}$ at the same time, see Tables 2 and 3) are employed to validate the effectiveness of Theorem 13.

In this section, all the numerical examples and simulation results share the same dynamics and initial function as those in Section 4.1 (that is the multiagent systems (3) with parameters (36) and initial function (38)).

In Table 2, the parameter matrix $\mathbf{K}_{1}$ is determined as

$$
\mathbf{K}_{1}=\left[\begin{array}{ccc}
3 & 1 & 1 \\
-5 & -1 & -2
\end{array}\right] \text {. }
$$

As we can see in this table, for undirected topology $\mathscr{G}_{b}$, comparisons are made between [28, Theorem 3] and our Theorem 10. Taking the situation $\mu=0.9$ as an example, the allowable upper bound on communication delay when designing a feasible $\mathbf{K}_{2}$ in [28, Theorem 3] is $h=0.026$, and the protocol parameter matrix is

$$
\mathbf{K}_{2}=\left[\begin{array}{ccc}
0 & 0 & 0.0866 \\
-0.0020 & 0.2230 & 0
\end{array}\right] .
$$

However, in our Theorem 10, the allowable upper bound is $h=0.295$, which increases one order of magnitude than that in [28, Theorem 3]. The protocol parameter matrix is

$$
\mathbf{K}_{2}=\left[\begin{array}{ccc}
0 & 0 & 0.254 \\
-0.612 & 1.031 & 0
\end{array}\right] \text {, }
$$

and the effectiveness is validated by simulations in Figure 3 .

Similarly, for directed topology $\mathscr{G}_{a}$, the comparisons between [28, Theorem 3] and our Theorem 13 are made in 
TABLE 2: Protocol parameter $\mathbf{K}_{2}$ designs for third-order linear multiagent systems with topologies $\mathscr{G}_{a}, \mathscr{G}_{b}$ in different $h$ and $\mu$.

\begin{tabular}{|c|c|c|c|c|c|c|c|c|c|c|}
\hline \multirow{3}{*}{28, Theorem 3] $\mathscr{G}_{b}$} & \multirow[b]{2}{*}{$\mathbf{K}_{2}$} & \multicolumn{3}{|c|}{$\mu=0.5$} & \multicolumn{3}{|c|}{$\mu=0.9$} & \multicolumn{3}{|c|}{ Unknown $\mu$} \\
\hline & & {$\left[\begin{array}{c}0 \\
-2.7589\end{array}\right.$} & $\begin{array}{c}0 \\
66.7789\end{array}$ & $\begin{array}{c}-5.7868 \\
0\end{array}$ & {$\left[\begin{array}{c}0 \\
-0.0020\end{array}\right.$} & $\begin{array}{c}0 \\
0.2230\end{array}$ & $\begin{array}{c}0.0866 \\
0\end{array}$ & \multicolumn{3}{|c|}{-} \\
\hline & $h$ & \multicolumn{3}{|c|}{0.112} & \multicolumn{3}{|c|}{0.026} & \multicolumn{3}{|c|}{-} \\
\hline \multirow[t]{2}{*}{ Theorem $10 \mathscr{G}_{b}$} & $\mathbf{K}_{2}$ & {$\left[\begin{array}{c}0 \\
-0.622\end{array}\right.$} & $\begin{array}{cc} & 0 \\
2 & 1.038\end{array}$ & $\begin{array}{c}0.260 \\
0\end{array}$ & {$\left[\begin{array}{c}0 \\
-0.612\end{array}\right.$} & $\begin{array}{c}0 \\
1.031\end{array}$ & $\begin{array}{c}0.254 \\
0\end{array}$ & {$\left[\begin{array}{c}0 \\
-0.617\end{array}\right.$} & $\begin{array}{c}0 \\
1.032\end{array}$ & $\begin{array}{c}0.277 \\
0\end{array}$ \\
\hline & $h$ & \multicolumn{3}{|c|}{0.294} & \multicolumn{3}{|c|}{0.295} & \multicolumn{3}{|c|}{0.295} \\
\hline \multirow[t]{2}{*}{28, Theorem 3] $\mathscr{G}_{a}$} & $\mathbf{K}_{2}$ & {$\left[\begin{array}{c}0 \\
-0.0007\end{array}\right.$} & $\begin{array}{c}0 \\
0.0357\end{array}$ & $\begin{array}{c}-0.0062 \\
0\end{array}$ & \multicolumn{3}{|c|}{-} & \multicolumn{3}{|c|}{-} \\
\hline & $h$ & \multicolumn{3}{|c|}{0.064} & \multicolumn{3}{|c|}{-} & \multicolumn{3}{|c|}{-} \\
\hline \multirow[t]{2}{*}{ Theorem $13 \mathscr{G}_{a}$} & $\mathbf{K}_{2}$ & {$\left[\begin{array}{c}0 \\
-1.1978\end{array}\right.$} & $\begin{array}{c}0 \\
2.0811\end{array}$ & $\begin{array}{c}0.7448 \\
0\end{array}$ & {$\left[\begin{array}{c}0 \\
-1.3319\end{array}\right.$} & $\begin{array}{c}0 \\
1.9809\end{array}$ & $\begin{array}{c}0.4469 \\
0\end{array}$ & {$\left[\begin{array}{c}0 \\
-1.3319\end{array}\right.$} & $\begin{array}{c}0 \\
1.9809\end{array}$ & $\begin{array}{c}0.4469 \\
0\end{array}$ \\
\hline & $h$ & & 0.050 & & & 0.047 & & & 0.047 & \\
\hline
\end{tabular}

TABLE 3: Protocol parameters $\mathbf{K}_{1}, \mathbf{K}_{2}$ designs for third-order linear multiagent systems with topologies $\mathscr{G}_{a}, \mathscr{G}_{b}$ in different $h$ and $\mu$.

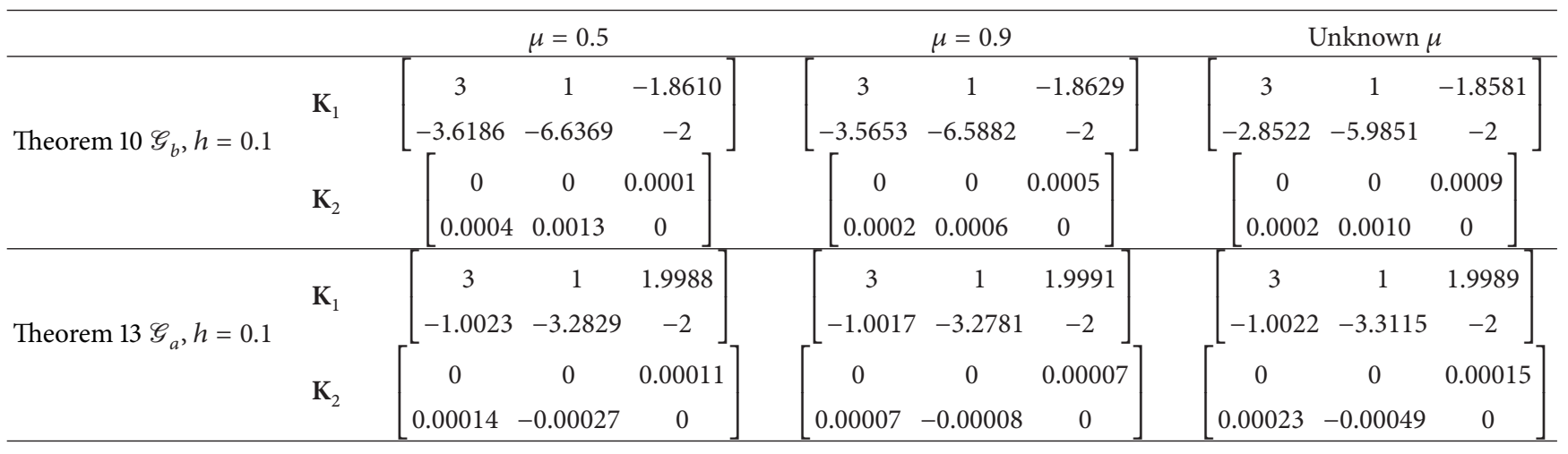

Table 2; while it should be noted that in the situation $\mu=0.5$, the allowable upper bound on communication delay in our Theorem 13 is $h=0.050$, which is less than that in [28, Theorem 3] $(h=0.064)$. However, this just holds in the case that $\mu$ is small. When $\mu$ becomes $0.9,[28$, Theorem 3$]$ cannot find a feasible $\mathbf{K}_{2}$ any more (actually, the allowable upper bound on $\mu$ for [28, Theorem 3] is 0.766). Correspondingly, we can see that the allowable upper bound for [28, Theorem 3] under communication topology $\mathscr{G}_{b}$ experiences a dramatic decrease when $\mu$ becomes larger. Therefore, [28, Theorem 3] is not robust enough for $\mu$. By contrast, $h$ in our Theorem 13 is relatively stable when $\mu$ varies. For $\mathscr{G}_{b}$, our Theorem 13 calculates the upper bound for communication delay $h=$ 0.294 in the situation $\mu=0.5$, while $h=0.295$ in $\mu=0.9$. It seems unreasonable, but it does happen when solving the NLMIs (24) and (25). This is because the CCL algorithm is not globally optimal.

Table 3 gives the information about the results for $\mathbf{K}_{1}, \mathbf{K}_{2}$ designs under undirected communication topology $\mathscr{G}_{b}$ (using Theorem 10) and directed communication topology $\mathscr{G}_{a}$ (using Theorem 13), respectively. It should be noted that $\mathbf{K}_{1}$ and $\mathbf{K}_{2}$ are calculated at the same time rather than $\mathbf{K}_{1}$ is determined in advance. To validate the effectiveness of our algorithms, we select the item with $h=0.1, \mu=0.9$ to carry on the simulation, and

$$
\begin{aligned}
\mathbf{K}_{1} & =\left[\begin{array}{ccc}
3 & 1 & 1.9991 \\
-1.0017 & -3.2781 & -2
\end{array}\right], \\
\mathbf{K}_{2} & =\left[\begin{array}{ccc}
0 & 0 & 0.00007 \\
0.00007 & -0.00008 & 0
\end{array}\right] .
\end{aligned}
$$

Figure 4 shows the effectiveness in our results with timevarying delay $\tau(t)=0.1 / 2+(0.1 / 2) \sin ((2 \times 0.9 / 0.1) t)$.

\section{Conclusions}

We investigated the consensus problems for high-order linear multiagent systems with time varying delays, which include a consensus convergence criterion and two protocol parameters design methods (for undirected and directed communication topologies, resp.). Mainly, the following contributions were concluded in this paper:

(1) a high-order linear multiagent systems consensus convergence criterion is proposed. By optimizing the structure on Lyapunov-Krasovskii functional and 
adding proper free-weighting matrices, the criterion has less conservativeness than the existing results;

(2) the consensus convergence criterion is validated when $\mu \geq 1$, and even can apply with a unknown $\mu$; while [28] could only fit for $\mu<1$;

(3) the protocol parameters design algorithm can give the two parameters, directly while the algorithm in [28] could only give $\mathbf{K}_{2}$ but the undetermined $\mathbf{K}_{1}$ would be set in advance;

(4) under the circumstance that the $\mathbf{K}_{1}$ is determined in advance, our theorems have less conservativeness and more robustness when the derivative of communication delay varies.

However, for the parameters designing part, the structures of matrices $\mathbf{V}_{1}, \mathbf{V}_{2}$ are constrained diagonal forms for the sake of eliminating some nonlinear inequality constraints. This has already brought some conservativeness because some feasible solutions for NLMIs are excluded manually. Therefore, the structure of $\mathbf{V}_{1}, \mathbf{V}_{2}$ will be investigated further so that our protocol parameters design could be better used in directed topologies with less conservativeness.

\section{References}

[1] W. Ren, R. W. Beard, and E. M. Atkins, "Information consensus in multivehicle cooperative control," IEEE Control Systems Magazine, vol. 27, no. 2, pp. 71-82, 2007.

[2] M. Cao, A. S. Morse, and B. D. O. Anderson, "Reaching a consensus in a dynamically changing environment: a graphical approach," SIAM Journal on Control and Optimization, vol. 47, no. 2, pp. 575-600, 2008.

[3] X. Wang, T. Liu, and J. Qin, "Second-order consensus with unknown dynamics via cyclic-small-gain method," IET Control Theory \& Applications, vol. 6, no. 18, pp. 2748-2756, 2012.

[4] J. Lin, A. S. Morse, and B. D. O. Anderson, "The multi-agent rendezvous problem. II. The asynchronous case," SIAM Journal on Control and Optimization, vol. 46, no. 6, pp. 2120-2147, 2007.

[5] R. Olfati-Saber, "Flocking for multi-agent dynamic systems: algorithms and theory," IEEE Transactions on Automatic Control, vol. 51, no. 3, pp. 401-420, 2006.

[6] C.-L. Liu and Y.-P. Tian, "Formation control of multi-agent systems with heterogeneous communication delays," International Journal of Systems Science, vol. 40, no. 6, pp. 627-636, 2009.

[7] R. Olfati-Saber, "Distributed Kalman filter with embedded consensus filters," in Proceedings of the 44th IEEE Conference on Decision and Control, and the European Control Conference (CDC-ECC '05), pp. 8179-8184, Seville, Spain, December 2005.

[8] R. Olfati-Saber and R. M. Murray, "Consensus problems in networks of agents with switching topology and time-delays," IEEE Transactions on Automatic Control, vol. 49, no. 9, pp. 15201533, 2004.

[9] X. Liu, W. Lu, and T. Chen, "Consensus of multi-agent systems with unbounded time-varying delays," IEEE Transactions on Automatic Control, vol. 55, no. 10, pp. 2396-2401, 2010.

[10] U. Münz, A. Papachristodoulou, and F. Allgöwer, "Consensus in multi-agent systems with coupling delays and switching topology," IEEE Transactions on Automatic Control, vol. 56, no. 12, pp. 2976-2982, 2011.
[11] P. Lin, K. Qin, H. Zhao, and M. Sun, "A new approach to average consensus problems with multiple time-delays and jointlyconnected topologies," Journal of the Franklin Institute, vol. 349, no. 1, pp. 293-304, 2012.

[12] Y. G. Sun, L. Wang, and G. Xie, "Average consensus in networks of dynamic agents with switching topologies and multiple timevarying delays," Systems \& Control Letters, vol. 57, no. 2, pp. 175$183,2008$.

[13] F. Xiao and L. Wang, "Asynchronous consensus in continuoustime multi-agent systems with switching topology and timevarying delays," IEEE Transactions on Automatic Control, vol. 53, no. 8, pp. 1804-1816, 2008.

[14] J. Qin, H. Gao, and W. X. Zheng, "On average consensus in directed networks of agents with switching topology and time delay," International Journal of Systems Science, vol. 42, no. 12, pp. 1947-1956, 2011.

[15] Y. G. Sun and L. Wang, "Consensus problems in networks of agents with double-integrator dynamics and time-varying delays," International Journal of Control, vol. 82, no. 10, pp. 19371945, 2009.

[16] Y. Gao and L. Wang, "Asynchronous consensus of continuoustime multi-agent systems with intermittent measurements," International Journal of Control, vol. 83, no. 3, pp. 552-562, 2010.

[17] J. Hu and Y. S. Lin, "Consensus control for multi-agent systems with double-integrator dynamics and time delays," IET Control Theory \& Applications, vol. 4, no. 1, pp. 109-118, 2010.

[18] P. Lin and Y. Jia, "Consensus of a class of second-order multiagent systems with time-delay and jointly-connected topologies," IEEE Transactions on Automatic Control, vol. 55, no. 3, pp. 778-784, 2010.

[19] F. Xiao, L. Wang, and J. Chen, "Partial state consensus for networks of second-order dynamic agents," Systems \& Control Letters, vol. 59, no. 12, pp. 775-781, 2010.

[20] K. Liu, G. Xie, and L. Wang, "Consensus for multi-agent systems under double integrator dynamics with time-varying communication delays," International Journal of Robust and Nonlinear Control, vol. 22, no. 17, pp. 1881-1898, 2012.

[21] G. Wen, Z. Duan, W. Yu, and G. Chen, "Consensus of secondorder multi-agent systems with delayed nonlinear dynamics and intermittent communications," International Journal of Control, vol. 86, no. 2, pp. 322-331, 2013.

[22] J. Qin, H. Gao, and W. X. Zheng, "Second-order consensus for multi-agent systems with switching topology and communication delay," Systems \& Control Letters, vol. 60, no. 6, pp. 390-397, 2011.

[23] J. Qin and H. Gao, "A sufficient condition for convergence of sampled-data consensus for double-integrator dynamics with nonuniform and time-varying communication delays," IEEE Transactions on Automatic Control, vol. 57, no. 9, pp. 2417-2422, 2012.

[24] Q. Zhang, Y. Niu, L. Wang, L. Shen, and H. Zhu, "Average consensus seeking of high-order continuous-time multi-agent systems with multiple time-varying communication delays," International Journal of Control, Automation and Systems, vol. 9, no. 6, pp. 1209-1218, 2011.

[25] U. Münz, A. Papachristodoulou, and F. Allgöwer, "Delay robustness in consensus problems," Automatica, vol. 46, no. 8, pp. $1252-1265,2010$.

[26] Y.-P. Tian and Y. Zhang, "High-order consensus of heterogeneous multi-agent systems with unknown communication delays," Automatica, vol. 48, no. 6, pp. 1205-1212, 2012. 
[27] Y. Zhang and Y. P. Tian, "Allowable delay bound for consensus of linear multi-agent systems with communication delay," International Journal of Systems Science, 2013.

[28] J. Xi, Z. Shi, and Y. Zhong, "Consensus analysis and design for high-order linear swarm systems with time-varying delays," Physica A, vol. 390, no. 23-24, pp. 4114-4123, 2011.

[29] M. Wu, Y. He, and J.-H. She, Stability Analysis and Robust Control of Time-Delay Systems, Science Press, Beijing, China and Springer, Berlin, Germany, 2010.

[30] L. El Ghaoui, F. Oustry, and M. AitRami, "A cone complementarity linearization algorithm for static output-feedback and related problems," IEEE Transactions on Automatic Control, vol. 42, no. 8, pp. 1171-1176, 1997.

[31] Y. He, G. P. Liu, D. Rees, and M. Wu, "Improved stabilisation method for networked control systems," IET Control Theory and Applications, vol. 1, no. 6, pp. 1580-1585, 2007. 


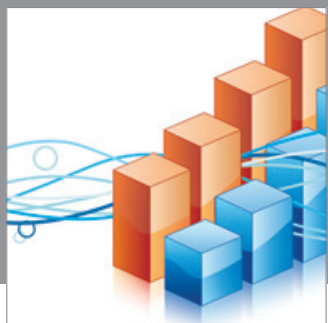

Advances in

Operations Research

mansans

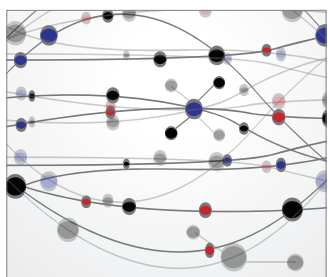

The Scientific World Journal
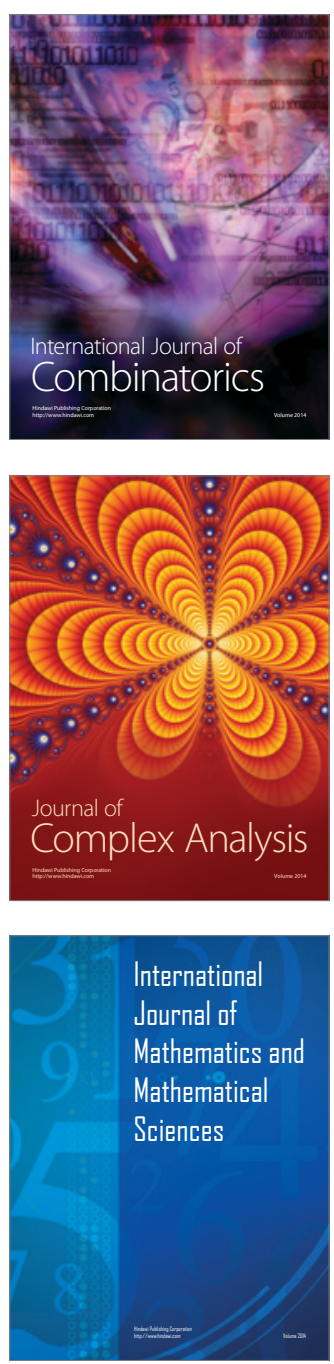
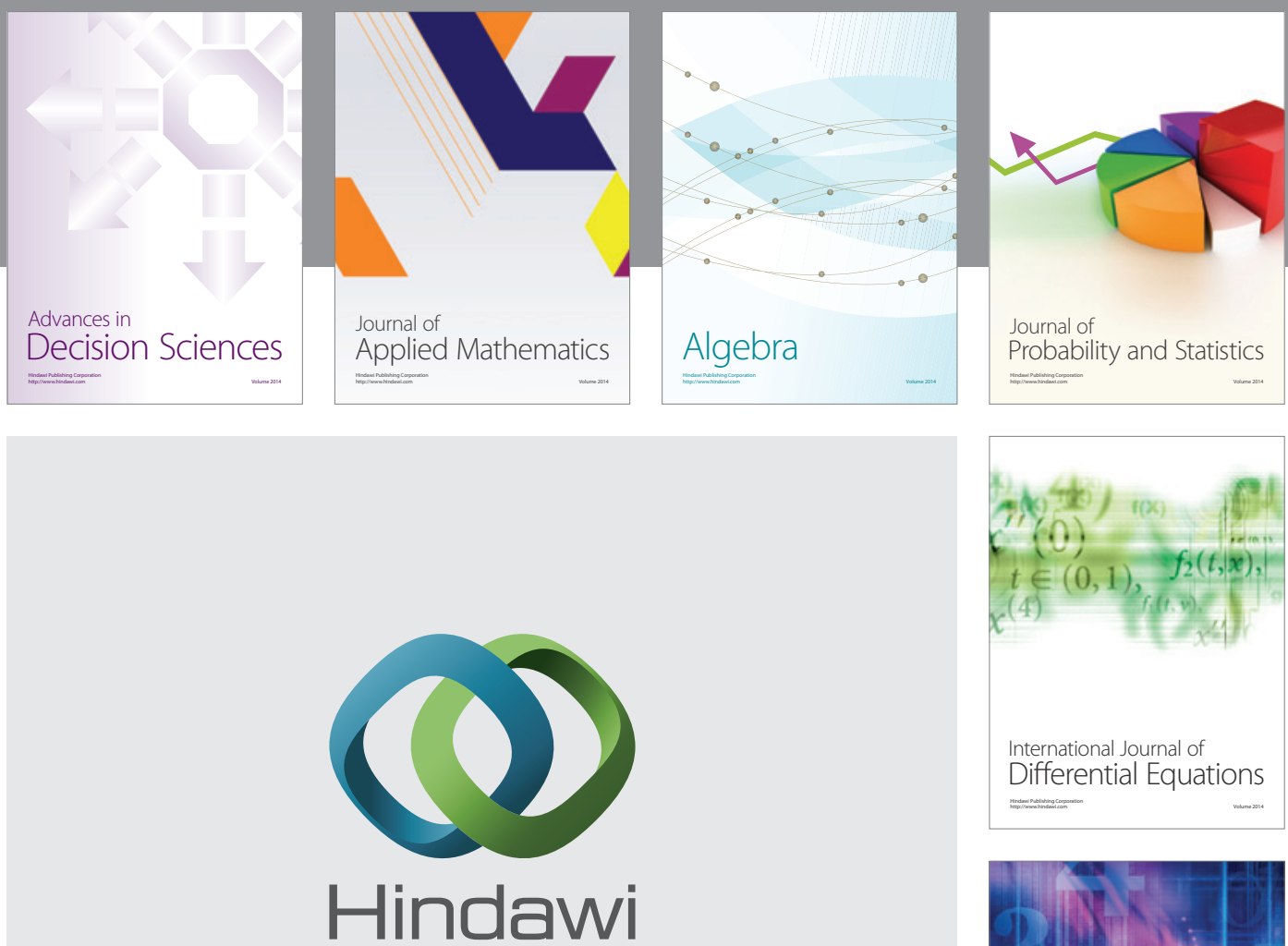

Submit your manuscripts at http://www.hindawi.com
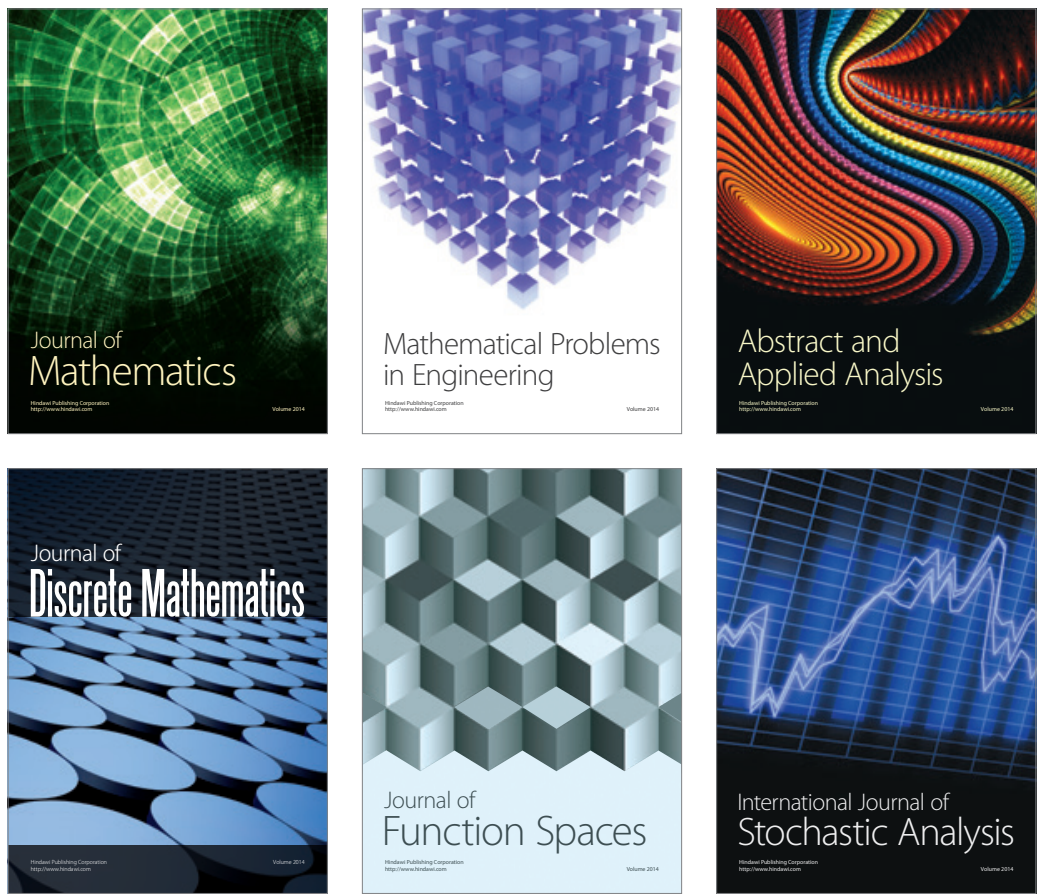

Journal of

Function Spaces

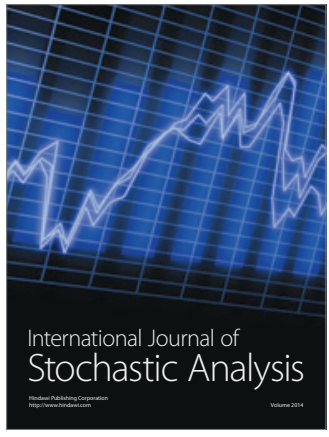

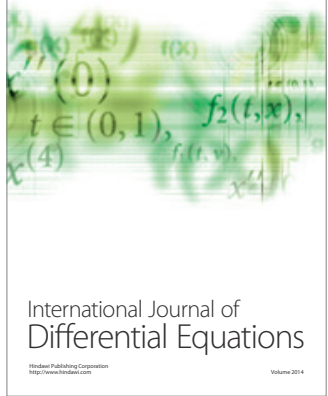
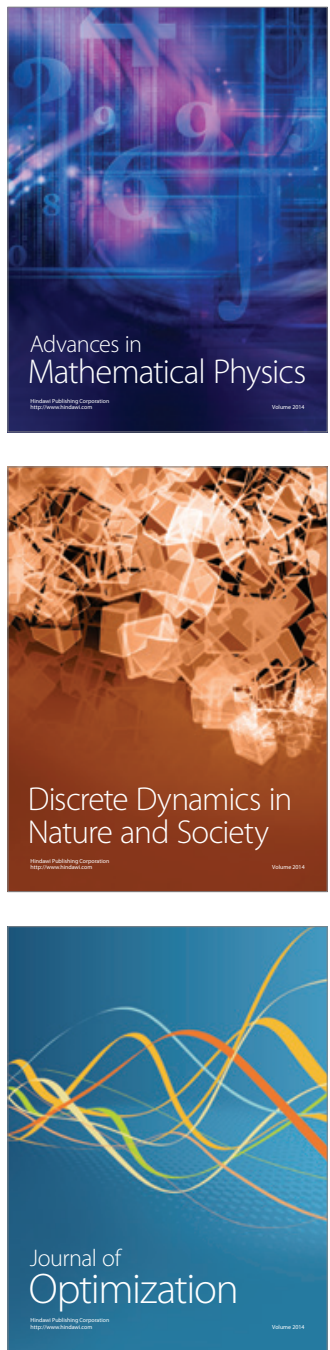